\title{
LOS REPRESENTANTES IBÉRICOS DE LA TRIBU LYTTINI (COLEOPTERA, MELOIDAE)
}

\author{
M. García-París ${ }^{1 *}$, J. L. Ruiz ${ }^{2}$ y M. París ${ }^{1}$
}

\begin{abstract}
RESUMEN
En este trabajo se revisa la distribución geográfica de las especies ibéricas de la tribu Lyttini (Meloidae). Los 1955 ejemplares estudiados corresponden a seis especies distribuidas en cinco géneros: Berberomeloe, Lagorina, Lytta, Oenas y Physomeloe. Berberomeloe fue creado para B. majalis, una especie ibero-magrebí cuya morfología larvaria no deja lugar a dudas sobre su inclusión entre los Lyttini. El género Berberomeloe cuenta con una segunda especie ibérica, $B$. insignis, recientemente rehabilitada y endémica de una estrecha área en el sureste ibérico. El género Lagorina incluye dos especies de la región mediterránea occidental, una de ellas, L. sericea, se encuentra en el sur peninsular y el noroeste de África. El género Lytta comprende cerca de 120 especies de las que únicamente L. vesicatoria se encuentra en Europa Occidental. A pesar de que ha sido repetidamente citada de toda la Península Ibérica, la mayoría de los ejemplares examinados se localizan al norte del Sistema Central. El género Oenas se ha venido considerando representado en la Península Ibérica por tres especies: $O$. afer, $O$. fusicornis y $O$. crassicornis. Todos los individuos ibéricos examinados corresponden morfológicamente a $O$. fusicornis, por lo que las citas ibéricas de $O$. afer probablemente se deban a confusiones con esa especie. El género Physomeloe incluye una única especie endémica de la Península Ibérica, . corallifer, bien distribuida por el centro de Portugal y la meseta española con citas escasas en otras áreas peninsulares.
\end{abstract}

Palabras clave: Coleoptera, Meloidae, Lyttini, Península Ibérica, Faunística, Corología.

\begin{abstract}
Iberian taxa of the tribe Lyttini (Coleoptera: Meloidae)

We revise the geographic distribution of the Iberian species of the tribe Lyttini (Meloidae). The 1955 specimens studied correspond to six species, distributed in five genera: Berberomeloe, Lagorina, Lytta, Oenas, and Physomeloe. Berberomeloe was erected for B. majalis, a species distributed in the Iberian Peninsula and northern Africa, whose larval morphology clearly supports its inclusion within the Lyttini. Berberomeloe presents a second species recently resurected taxonomically, B. insignis, endemic to the southeastern regions of Iberia. Lagorina includes two species from the western Mediterranean Region, one of which, L. sericea, is found in the southern regions of Iberia and northern Africa. Lytta is represented by more than 120 species, but only one, L. vesicatoria, is present in western Europe. The species was often cited from all over the Iberian Peninsula, but most of the specimens examined are located north of the Sistema Central. The genus Oenas was considered to be represented in the Iberian Peninsula by three species: $O$. afer, $O$. fusicornis y $O$. crassicornis. All specimens examined correspond morphologically to $O$. fusicornis, so the Iberian records of $O$. afer probably are misidentifications. Physomeloe includes a single species, $P$. corallifer, which is endemic to the Iberian Peninsula. It is well distributed in central Portugal and the Spanish Plateau with scarce records in other Iberian regions.
\end{abstract}

Key words: Coleoptera, Meloidae, Lyttini, Iberian Peninsula, Faunistics, Corology.

\footnotetext{
Museo Nacional de Ciencias Naturales. CSIC. José Gutiérrez Abascal, 2. 28006, Madrid. España.

Parques de Ceuta, I, $3^{\circ}$ D, 51002 Ceuta. España.

Autor para correspondencia
} 


\section{Introducción}

Aunque la fauna de meloidos ibéricos puede considerarse relativamente bien conocida a nivel general (Górriz Muñoz, 1882; Rodríguez LópezNeyra, 1914; Fuente, 1933; Pardo Alcaide, 1949; 1950; 1956; 1958; Ruiz \& Avila, 1993; Ruiz et al., 1993, 1994a, 1994b; Ruiz, 1996, 2001; Serrano, 1983; García-París, 2000; Capote \& García-París, 2001; Trotta \& García-París, 2001; Jeremías et al., 2002; Recalde et al., 2002) apenas existen datos precisos a nivel local o regional sobre la distribución geográfica de las especies y en algunos casos, existen problemas taxonómicos o errores de identificación arrastrados a través de la bibliografía que dificultan la atribución de las citas a unas especies u otras (García-París, 1998; García-París et al., 1999; Ruiz, 2001; Recalde et al., 2002).

En los últimos años la sistemática de los meloidos ha sufrido profundas reorganizaciones basadas fundamentalmente en el examen de la morfología larvaria (Pinto \& Selander, 1970; Bologna, 1989; 1991; Bologna y Pinto, 2001, 2002). Entre las principales consecuencias de dichas reorganizaciones destaca la fragmentación del género Meloe y la inclusión de algunos de los géneros resultantes de dicha subdivisión en la tribu Lyttini LeConte, 1862. En lo tocante a la fauna ibérica, dos especies de carralejas tradicionalmente incluidas en el género Meloe han sido transferidas a la tribu Lyttini con el correspondiente cambio genérico: Berberomeloe majalis y Physomeloe corallifer (Bologna, 1989; Bologna \& Aloisi, 1994). Siguiendo estos cambios la tribu Lyttini estaría representada en la Península Ibérica por cinco géneros: Berberomeloe, Lagorina, Lytta, Oenas y Physomeloe.

Los únicos trabajos que aportan datos a nivel general sobre la distribución de Lyttini ibéricos son los de Górriz Muñoz (1882), Oliveira (1894), Rodríguez López-Neyra (1914), Fuente (1933) y Pardo Alcaide (1950). En estos trabajos se presentan distribuciones generalizadas para la mayoría de las especies, sin precisar localidades concretas. Bologna (1989), Bologna \& Aloisi (1994), García-París (1998) y García-París et al. (1999) revisan la distribución geográfica ibérica y taxonomía de Berberomeloe y Physomeloe. Exceptuando los trabajos de Valladares \& Salgado (1983) y Valladares (1984) sobre los meloidos de la provincia de León, el listado de Pérez Moreno \& Cabrero Sañudo (1999) de La Rioja, y la revisión de los Meloidae de Aragón (Recalde et al., 2002), la mayoría de los trabajos a nivel regional únicamente recogen datos puntuales (Dieck, 1870; Salvañá Comas, 1870; Martínez Sáez,
1873; Uhagón, 1887; Cuní Martorell, 1888; Medina, 1895; Górriz Muñoz, 1902; Flach, 1907; Redondo, 1915; Sumakov, 1934; Cobos, 1949; Ruiz \& Avila, 1993; Ruiz et al. 1994a; Aguiar \& Serrano, 1995).

El examen e identificación del material de varias colecciones peninsulares nos ha proporcionado una abundante fuente de datos para precisar la distribución peninsular de las especies de esta tribu, determinar aspectos de su variabilidad morfológica y además, permitirnos discutir algunos problemas taxonómicos. Entre estos últimos destacan problemas como el de la posición taxonómica y validez específica de Berberomeloe insignis (García-París, 1998; García-París et al., 1999), o el del número de especies del género Oenas que ocupan la Península. En este trabajo presentamos los primeros datos de conjunto sobre la distribución de Lyttini ibéricos y discutimos algunos de los problemas taxonómicos indicados.

\section{Material y método}

El material estudiado incluye 1955 ejemplares de las colecciones del Museo Nacional de Ciencias Naturales, C.S.I.C., Madrid (MNCN); del Museu de Zoologia de Barcelona (MZB), de la Universidad de Alicante (UA), de la Universidad Politécnica de Évora (UPE), de la Universidad de Salamanca (USal), de la Estación Experimental de Zonas Áridas, C.S.I.C., Almería (EEZA), de la Sociedad Andaluza de Entomología, Córdoba (SAE), y de la colecciones privadas de A. J. Zuzarte, Monforte (AJZ), J. L. Zapata, Madrid (JLZ), J. L. Ruiz, Ceuta (JLR), J. de Ferrer, Algeciras (JF), M. Baena, Córdoba (MB), y de M. A. Sobrino, Madrid (MAS). Muchos de los ejemplares examinados proceden de las colecciones históricas de L. Pérez Arcas, M. M. de la Escalera, C. Bolívar y otros conocidos entomológos españoles. Algunos de estos ejemplares ya fueron citados en obras previas (Rodríguez López-Neyra, 1914; Fuente, 1933), pero exceptuando los casos de Physomeloe y Berberomeloe examinados respectivamente por Bologna \& Aloisi (1994) y GarcíaParís (1998), hasta ahora no se había efectuado una revisión crítica de los mismos. Además del material de colecciones se han estudiado ejemplares vivos o conservados en alcohol procedentes de muestreos efectuados por los autores en áreas con escaso nivel de prospección (provincias de Zamora, Cuenca, Teruel, Ciudad Real, Albacete) o en áreas dónde se requería la confirmación de citas antiguas (Almería, Madrid). Para la realización de los mapas 
se han buscado las UTM de 10x10 km de las localidades citadas. En muchos casos un único registro puede corresponder a más de una cuadrícula de $10 x 10 \mathrm{~km}$, en esos casos se ha seleccionado una de las posibles, en general aquella que incluye la mayor parte del municipio o lleva el nombre del accidente geográfico en cuestión. En los mapas, para permitir una visualización adecuada, se presentan los puntos en coordenadas UTM de 20 x 20 $\mathrm{km}$ lo que reduce considerablemente el número total de puntos. La clasificación adoptada sigue a Bologna (1991).

\section{Resultados y discusión}

Los ejemplares estudiados corresponden a seis especies distribuidas en los cinco géneros habitualmente citados en la Península. Los géneros Alosimus Mulsant, 1857 y Cabalia Mulsant \& Rey, 1858, representados respectivamente por $A$. collaris (Fabricius, 1787) y C. segetum (Fabricius, 1792) en las claves de Rodríguez López-Neyra (1914), han de excluirse de la fauna ibérica mientras su presencia no sea documentada. La presencia de $C$. segetum en la Península se debe a una cita de Pérez Arcas en Andalucía, que Górriz Muñoz (1882) y Rodríguez López-Neyra (1914) recogen en sus trabajos.

Los géneros peninsulares pueden identificarse con la siguiente clave artificial:

1. Élitros con los márgenes suturales divergentes, imbricados en la base, en general mucho más cortos que el abdomen.

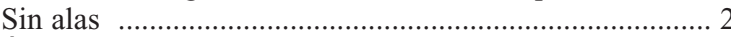

- Élitros con los márgenes suturales paralelos, no imbricados, generalmente tan largos como el abdomen. Alados ....

2. Protorax con un par de protuberancias laterales de color rojo en cada lado. Antenas con el último artejo no bifurcado. Coloración general negra ........................ Physomeloe

Protorax subcuadrangular sin protuberancias laterales. Antenas con el último artejo claramente bifurcado. Coloración general negra, aunque con frecuencia presentan una banda transversal anaranjada en el dorso de cada segmento abdominal, o alternativamente manchas anaranjadas en las sienes ............................................. Berberomeloe

3. Antenas más cortas que la cabeza y el pronoto juntos. Lóbulo superior de las uñas con el margen inferior dentado. Coloración tegumentaria nunca verde metálica

Oenas

- Antenas más largas que la cabeza y el pronoto juntos. Lóbulo superior de las uñas con el margen inferior sin dientes. Coloración tegumentaria verde metálica ............ 4

4. Cabeza profundamente surcada en la línea media. Pronoto con ángulos anteriores marcados ............................ Lytta

- Cabeza sin surco en en la línea media. Pronoto con ángulos anteriores redondeados

\section{Berberomeloe Bologna,1989}

El género Berberomeloe fue creado por Bologna (1989) para B. majalis (Linnaeus, 1758), una especie ibero-magrebí tradicionalmente incluida en el género Meloe por sus caracteristicas morfológicas externas, pero cuya morfología larvaria no deja lugar a dudas sobre su inclusión entre los Lyttini. García-París (1998) incluye una segunda especie en el género, $B$. insignis, exclusiva de una estrecha franja del sureste ibérico. Las especies del género Berberomeloe pueden separarse según la clave siguiente:

1. Cabeza con las sienes prominentes, rojo-anaranjadas y a menudo con una mancha rojiza frontal. Puntuación cefálica ausente o muy fina. Machos con los artejos antenales alargados con un diente muy desarrollado en los artejos $\mathrm{V}$, VII y IX . Pronoto con los ángulos anteriores muy salientes, y lados convergentes posteriormente. Coloración general negra excepto en la región cefálica ............ B. insignis

- Cabeza con las sienes redondeadas, de color totalmente negro. Si existe una mancha frontal rojiza es redondeada. Puntuación cefálica generalmente muy densa o espaciada pero siempre marcada. Machos con los artejos antenales trapezoidales y con dientes externos poco marcados. Pronoto con los ángulos anteriores rectos, apenas salientes y lados paralelos. Coloración general variable, negra, o con más frecuencia con una banda transversal roja o anaranjada en la parte posterior de cada terguito abdominal B. majalis

\section{Berberomeloe insignis (Charpentier, 1818)}

Se trata de una especie endémica de una pequeña franja del sureste peninsular limitada a las provincias de Granada, Almería y Murcia (Fig. 1), que se caracteriza entre otros rasgos por su gran tamaño, por una morfología de los segmentos antenales peculiar, por presentar el pronoto con los ángulos anteriores muy salientes y por su coloración cefáfica. Las variaciones observadas corresponden a diferencias de talla $(12-49 \mathrm{~mm})$ y de pigmentación con una extensión variable de las manchas cefálicas, especialmente de la mancha frontal (GarcíaParís, 1998).

Además de los trabajos recientes de GarcíaParís (1998) y García-París et al. (1999) los únicos datos precisos publicados sobre su distribución son: Cartagena (Murcia) (Küster, 1847), Motril (Granada) (Rodríguez López-Neyra, 1914), y una fotografía de un ejemplar de las Dunas del Cabo de Gata (Almería) (Cardelús, 1987: 123). Los demás autores únicamente señalan las provincias de Murcia o Granada de forma general (Escherich, 


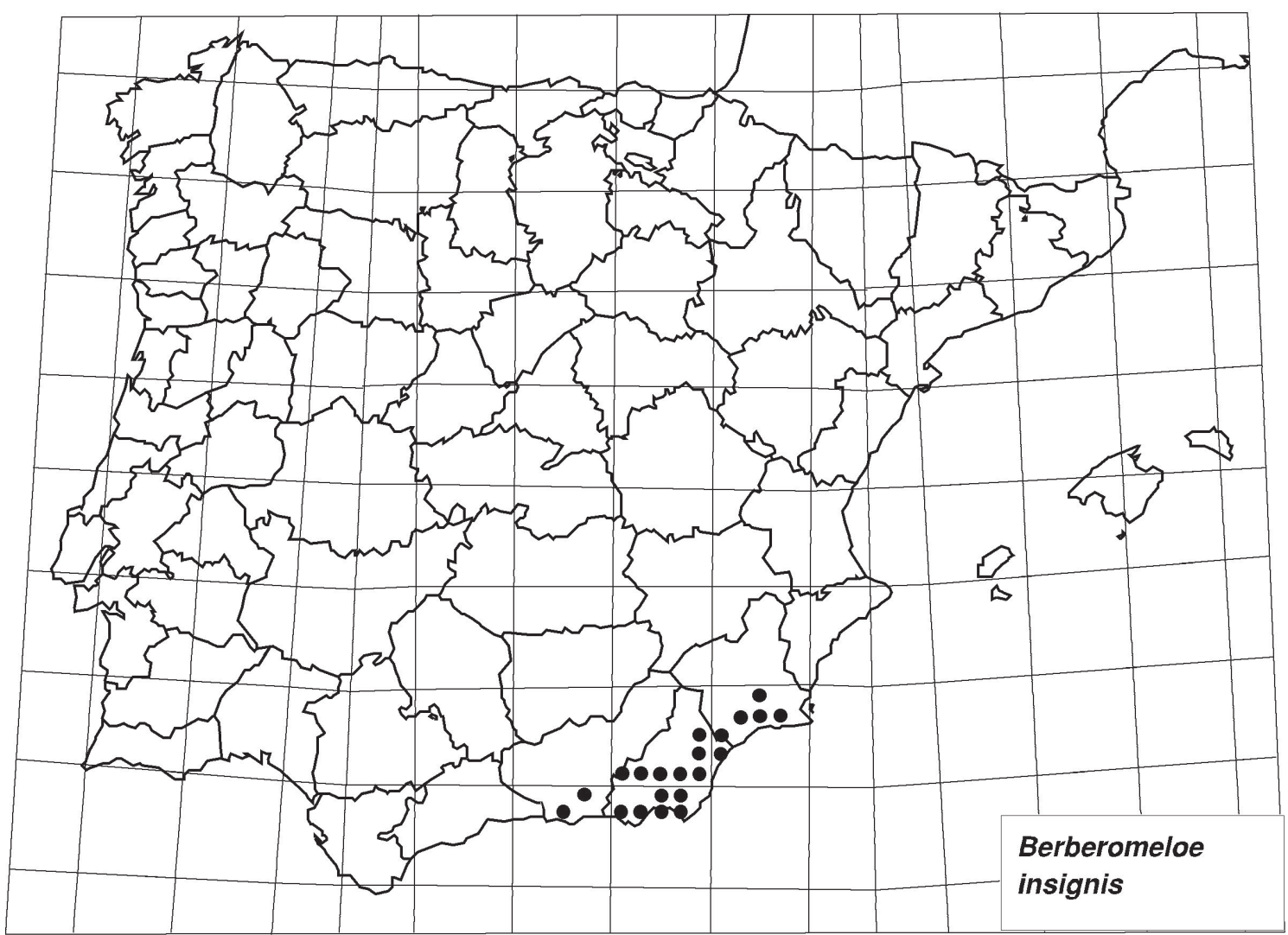

Fig. 1. - Mapa de la Península Ibérica con los puntos correspondientes a las localidades donde se ha localizado o citado en la bibliografía Berberomeloe insignis (Charpentier, 1818). Cada punto equivale a una cuadrícula UTM de 20 x $20 \mathrm{~km}$.

Fig. 1.- Map of the Iberian Peninsula with locality records for Berberomeloe insignis (Charpentier, 1818). Each dot represents a 20 x $20 \mathrm{~km}$ UTM square.

1896; Fuente, 1933). La cita correspondiente a un ejemplar de Moreda (Granada) publicada por García-París (1998) es errónea y se debe a un error de transcripción de la etiqueta, ya que, tras una comparación directa con etiquetas de otros ejemplares también colectados por S. de Uhagón de la misma procedencia (Pimelia), se confirma que éste ejemplar proviene de Morata (Murcia). Con esta rectificación se elimina la única cita de $B$. insignis de la Hoya de Guadix-Baza, donde la presencia de la especie ya había sido cuestionada (García-París et al., 1999). En la colección del MNCN existe un ejemplar con etiqueta de "Menorca" que consideramos un error de etiquetado.

Muchas de sus poblaciones se encuentran seriamente amenazadas por la utilización de productos fitosanitarios en las explotaciones agrícolas intensivas que ocupan gran parte de su limitada área de distribución, por lo que la especie en conjunto podría considerarse amenazada, aunque la falta actual de información sobre regresiones poblacionales o de su área de ocupación en las últimas décadas no permite realizar una evaluación adecuada de su estatus. Se ha encontrado en microsimpatría con B. majalis en las faldas de la Sierra de Filabres, entre Sierra Alhamilla y Sierra Cabrera, y en la Rambla Seca de Tabernas (Almería) (García-París et al., 1999). La fenología imaginal parece centrarse en los meses de marzo a mayo (García-París, 1998; García-París et al., 1999).

MATERIAL EXAMINADO: Se han revisado 31 ejemplares correspondientes en parte al material reseñado en García-París (1998) y García-París et al. (1999) y a los siguientes ejemplares: ESPAÑA: ALMERÍA: Pulpí: 14-IV-1999, 3 ejemplares (M. García París e I. Martínez Solano leg.) (MNCN). Sierra Alhamilla: 1-V-1986, 3 ejemplares (L. García leg.) (JLR). GRANADA: Órgiva: 21-V-1992, 1 ejemplar (J. A. Hernández leg.) (JLR). MURCIA: Morata: 1 ejemplar (Uhagón leg.) (MNCN). 


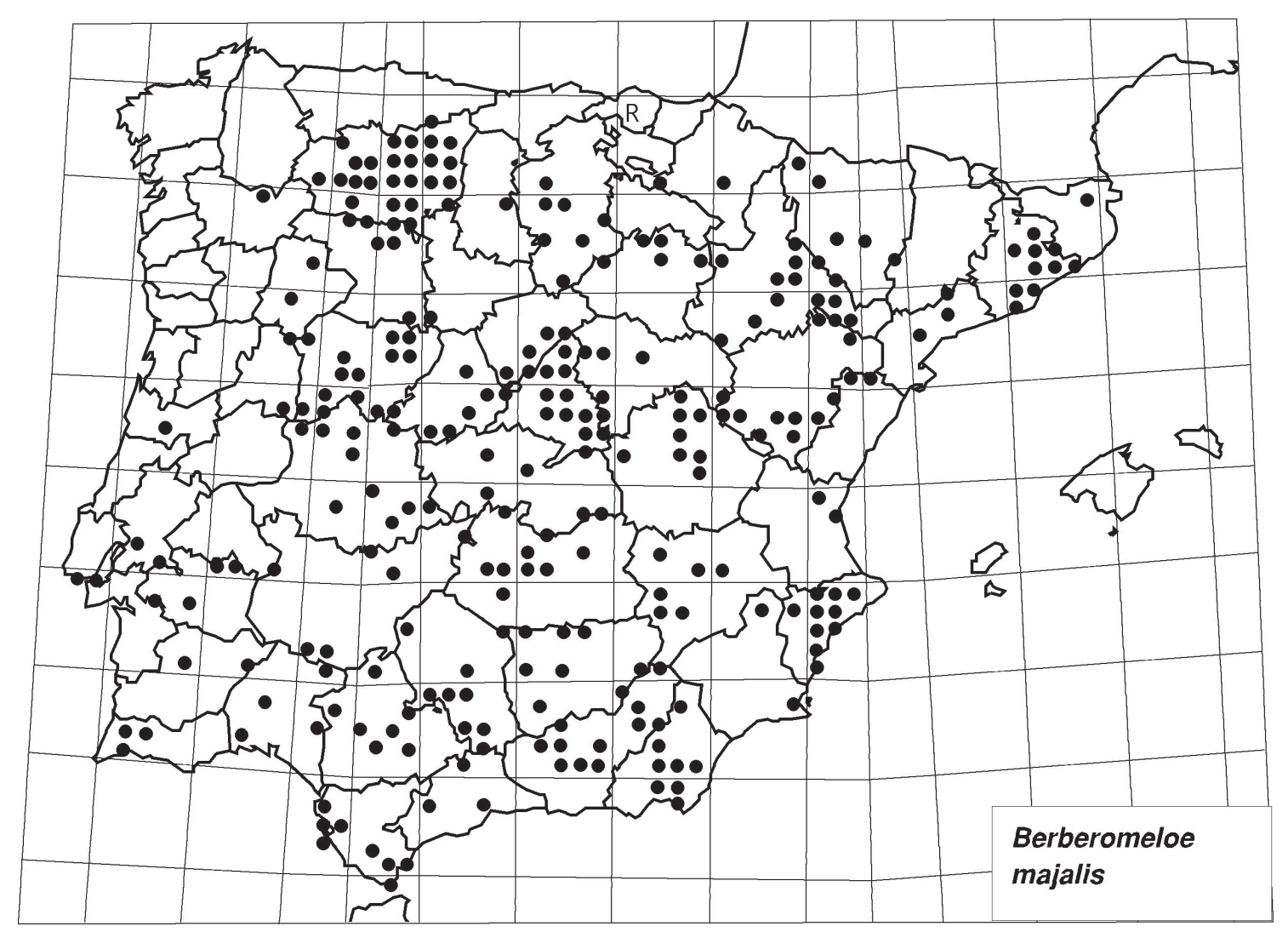

Fig. 2.- Mapa de la Península Ibérica con los puntos correspondientes a las localidades donde se han localizado o citado en la bibliografía ejemplares de Berberomeloe majalis (Linnaeus, 1758). Cada punto equivale a una cuadrícula UTM de 20 x $20 \mathrm{~km}$.

Fig. 2.- Map of the Iberian Peninsula with locality records for Berberomeloe majalis (Linnaeus, 1758). Each dot represents a 20 x $20 \mathrm{~km}$ UTM square.

Además se han efectuado observaciones de ejemplares no capturados en MURCIA: Parque Regional El Valle-Carrascoy: Majal Blanco: 22-V-2002, 2 ejemplares (J. Kohler).

El material examinado o citado por otros autores corresponde a las siguientes cuadrículas UTM de 10x10 km: España: Almería (WF16, WF26, WF36, WF47, WF49, WF57, WF66, WF67, WF68, WF69, WG11, WG30, WG40, WG71, WG80, WG82, WG94, XG13, XG14); Granada (VF56, VF68); Murcia (XG36, XG46, XG59, XG76) (Fig. 1).

\section{Berberomeloe majalis (Linnaeus, 1758)}

Especie extendida por el noroeste de África, la Península Ibérica y una estrecha franja del sur de Francia (Bologna, 1989).

De acuerdo con la procedencia de los ejemplares examinados es una especie presente en toda la región mediterránea peninsular, que alcanza por el norte las estribaciones de la Cordillera Cantábrica, el País Vasco y las cadenas Prepirenaicas de Huesca (Fig. 2). A pesar de que ha sido citada en numerosas localidades ibéricas (Dieck, 1870; Salvañá Comas, 1870; Martínez Sáez, 1873; Cuní Martorell \& Martorell Peña, 1876; Górriz Muñoz, 1882; Uhagón, 1887; Cuní Martorell, 1888; Oliveira, 1894; Medina, 1895; Cuní Martorell, 1897; Champion, 1902; Górriz Muñoz, 1902; Laguna, 1902; Champion, 1903, 1904; Rodríguez LópezNeyra, 1914; Redondo, 1915; Fuente, 1927, 1933; Sumakov, 1934; Cobos, 1949; Torres Sala, 1962; Valladares y Salgado, 1983; Mitter, 1984; Serrano, 1983; Valladares Díez, 1984; Bologna, 1989; Ruiz et al., 1994a; Aguiar \& Serrano, 1995; GarcíaParís, 1998; García-París et al., 1999; Melic \& Blasco-Zumeta, 1999; Pérez Moreno \& Cabrero Sañudo, 1999; Recalde et al., 2002) todavía no existen citas para muchas zonas donde la especie está sin duda presente. 
Según García-París (1998) dentro de la Península Ibérica pueden distinguirse dos grupos de poblaciones con caracteres morfólogicos diferenciales. Los ejemplares de las poblaciones localizadas al sur del Guadalquivir y en las porciones sudorientales de la Península, presentan el pronoto y la cabeza apenas punteados, casi lisos. Por el contrario las poblaciones de la Meseta y del norte y oeste peninsular, con independencia de la presencia o ausencia de bandas abdominales anaranjadas o rojas, se caracterizan por presentar el pronoto y cabeza fuertemente punteados y con fosetas que a menudo confluyen entre sí, sobre todo en el pronoto.

Dentro del grupo septentrional, la coloración predominante de las poblaciones occidentales es negra con una banda transversal corta rojo-anaranjada dorsal en la parte posterior de cada segmento abdominal. Los ejemplares de Cataluña, gran parte de Aragón, norte de la Comunidad Valenciana y porción nororiental de Castilla-La Mancha tienen las bandas anaranajado-rojizas mucho más largas de forma que en algunos casos rodean completamente el segmento abdominal. Adicionalmete hay pequeñas áreas geográficas donde todos los ejemplares examinados son de coloración uniformemente negra (Algarve, centro y noreste de Madrid). Dentro del grupo bético, todos los individuos examinados de Sierra Nevada presentan una coloración completamente negra, mientras que en Cádiz y Málaga la coloración dominante es negra con las bandas rojizas transversales cortas.

La coloración en vivo de la bandas varía desde el rojo fuerte al amarillo anaranjado, aunque estas variaciones también pueden presentarse a nivel local. La longitud de los ejemplares ibéricos es muy variable (de 8 a $51 \mathrm{~mm}$ ) y pueden alcanzar hasta 75 $\mathrm{mm}$ (Bologna, 1988). Entre los ejemplares de menor talla suelen aparecer individuos con los élitros más largos que el abdomen.

MATERIAL EXAMINADO: Se han examinado 586 ejemplares, incluido el material reseñado en García-París (1998) y GarcíaParís et al. (1999): ESPAÑA: ALBACETE: Alcaraz: 4-IV1993, 1 ejemplar (M.A.S. Sobrino leg.) (MAS). Bogarra, El Malojar: 8-III-1997, 1 ejemplar (M. París y E. Izquierdo leg.) (MNCN). Sierra del Agua: 11-V-1997, 1 ejemplar (E. Izquierdo leg.) (MNCN). ALMERÍA: Campohermoso: 26-II2001, 1 ejemplar (M. García París leg.) (MNCN). Los Alamicos, Sierra de María, 1400 m, 30SWG6870: 19-V-1992, 3 ejemplares (P. Barranco leg.) (JLR). Sierra Filabres: 5-VII40. (MZB). ALICANTE: Serra Alfaro, Fatxeca: 20-IV-1980, 1 ejemplar (R. Plá leg.) (MZB). $2 \mathrm{~km}$ al E de Castell de Castells: 24-VI-1998, 1 ejemplar (M. García París y C. Martín leg.) (MNCN). AVILA: Santa Cruz del Valle: 15-IV-1981, 1 ejemplar (JLZ). $10 \mathrm{~km}$ al S de Las Navas del Marqués: 10-V-1998, 5 ejemplares (M. García París y C. Martín leg.) (MNCN).
BADAJOZ: Fuente de Cantos: 16-IV-1987, 1 ejemplar (A. F. Serna leg.) (USal). BARCELONA: Balenyá: 6 ejemplares (Villarrubia leg.) (MZB); 23-III-1928, 1 ejemplar (Villarrubia leg.) (MZB); 28-III-1928, 1 ejemplar (MZB); 18-III-1930, 1 ejemplar (Villarrubia leg.) (MZB); 1-VI-1930, 1 ejemplar (MZB); 20-IV-1930, 4 ejemplares (Villarrubia leg.) (MZB); V1930, 2 ejemplares (Villarrubia leg.) (MZB); 6-VII-1933, 1 ejemplar (Villarrubia leg.) (MZB); V-1934, 1 ejemplar (Villarrubia leg.) (MZB). Barcelona: 1 ejemplar (F. Ferrer leg.)(MZB); 17-V-26, 1 ejemplar (Col. Español) (MZB). Barcelona, Tibidabo: 1 ejemplar (MZB). Castelleir [Castellar del Vallés]: IV-1935, 4 ejemplares (Museu) (MZB). Castelldefels: 19-VI-08, 2 ejemplares (F. Ferrer leg.) (MZB). Centelles: 12-V-1984, 1 ejemplar (Escolá leg.) (MZB). Can Puigantic, Collsuspina (Moianes B): 28-VI-81, 1 ejemplar (O. Escolá leg.) (MZB). Colluspina: 6-VI-1988, 1 ejemplar (De Gregorio leg.) (MZB). Collsuspina: V-1939, 1 ejemplar (Español leg.) (MZB). El Berenguer, Monistrol Caldera: 12VI-1968, 1 ejemplar (O. Escolá leg.) (MZB). Granollers, 1 ejemplar (MZB). Cca. Mines Sta. Coloma, Colluspina: 30-VI84, 2 ejemplares (O. Escolá leg.) (MZB). Cca. Mines Sta. Coloma Sasserra, Colluspina: 25-V-1986, 2 ejemplares (Escolá leg.) (MZB). Moià: 19-V-1985, 1 ejemplar (O. Escolá leg.) (MZB); 14-VI-1987, 1 ejemplar (O. Escolá leg.) (MZB). MoiàPuig Rodó, Moià: 9-VIII-86, 1 ejemplar (Escolá leg.) (MZB). Moya: 30-V-76, 1 ejemplar (F. Español leg.) (MZB). S. Cugat [San Cugat del Vallés]: 1 ejemplar (Col. Ferrer Dalmau) (MZB). S. Justo, Desvern, El Vallés: 19-IV-1939, 1 ejemplar (MZB). San Segimond, Barcelona, Montsen.: 29-VI-1942, 1 ejemplar (J. Mateu leg.) (MZB). Santa Coloma de Gramanet: 2 ejemplares (MZB). St. Pere Ferrerons, Moià: 18-IV-1993, 1 ejemplar (Escolá leg.) (MZB). Sta. Coloma Sasserra, Colluspina: 19-V-1984, 1 ejemplar (O. Escolá leg.) (MZB). Vich: 1 ejemplar (Zariquiey leg.) (MZB). BURGOS: Melgar [de Fernamental]: 10-VIII-1984, 1 ejemplar (L. A. Fuente leg.) (USal). Solano: 8-VII-2000, 1 ejemplar (I. Martínez Solano leg.) (MNCN). CÁCERES: Hervás: 18-V-1986, 1 ejemplar (L. Muñoz leg.) (USal); IV-1987, 1 ejemplar (B. Jovellanos leg.) (USal); 25-V-1987, 1 ejemplar (J. L. Sánchez leg.) (USal); 25V-1987, 1 ejemplar (P. O'Mullony leg.) (USal); 29-IV-1987, 1 ejemplar (M. J. Gómez leg.) (USal). Jaraiz: 30-IV-1987, 1 ejemplar (Hannemann leg.) (USal). Pantano Gabriel y Galán: 29-IV-1987, 1 ejemplar (M. B. Escudero leg.) (USal); 29-IV1987, 1 ejemplar (M. A. García leg.) (USal). Pto. S. [Puerto de San] Vicente: 2-VI-1966, 2 ejemplares (Esp. Neg. leg.) (MZB). Puertollano, $8 \mathrm{~km}$ al E de Cañamero: 13-V-2000, 1 ejemplar (M. García París leg.) (MNCN). Río Ruecas, Zorita: 25-IV1998, 3 ejemplares (E. Pérez Zapirain leg.) (MNCN). TrujilloPuerto de Miravete: 1-V-1986, 1 ejemplar (A. Zuzarte leg.) (AZ). Valcochero, Plasencia: 4-IV-1985, 1 ejemplar (I. Campo leg.) (USal). Valle del Jerte: 21-VII-1985, 1 ejemplar (P. Conejero leg.) (USal). CÁDIZ: Alcalá de los Gazules: 2 ejemplares (López leg.) (MZB). Algeciras: 21-IV-1921, 1 ejemplar (A. Codina leg.) (MZB); 21-IV-1921, 1 ejemplar (Novellas leg.) (MZB); 22-IV-1921, 6 ejemplares (Novellas leg.) (MZB); 23-IV-1921, 4 ejemplares (A. Codina leg.) (MZB); 24-IV1921, 2 ejemplares (Novellas leg.) (MZB); 24-IV-1921, 4 ejemplares (A. Codina leg.) (MZB). Jerez: 16-III-1985, 1 ejemplar (C. Martín Gago leg.) (USal). Laguna de Medina, Jerez: 27-III-1983, 1 ejemplar (J. de Ferrer leg.) (JF). San Roque: V-1973, 2 ejemplares (J. de Ferrer leg.) (JLR y JF). Tarifa: IV-71, 1 ejemplar (MZB). CIUDAD REAL: Ciudad 
Real: 1-IV-1989, 1 ejemplar (M.A.S. Sobrino leg.). (MAS). [Casas de] Torrecilla: 13-III-1994, 1 ejemplar (J. L. Zapata leg.) (JLZ). Picón: 13-IV-1986, 1 ejemplar (JLZ). Pozuelo de Calatrava: 2 ejemplares (La Fuente leg.) (MZB). Pozuelo de Cva. [Calatrava]: 27-IV-1986, 1 ejemplar (JLZ). Anchuras, Finca Rosalejo: 25-III-1997, 2 ejemplares (M. París y E. Izquierdo leg.) (MNCN). $1 \mathrm{~km}$ al NO de Valenzuela: 20-III1999, 1 ejemplar (M. García París y C. Martín leg.) (MNCN). $1 \mathrm{~km}$ al SO de Pozuelo de Calatrava: 20-III-1999, 1 ejemplar (M. García París y C. Martín leg.) (MNCN). 2 Km al NO de Corral de Calatrava: 1-IV-2000, 1 ejemplar (I. Martínez Solano y M. García París leg.) (MNCN). 7 km al NW de Corral de Calatrava: 1-IV-2000, 1 ejemplar (I. Martínez Solano y M. García París leg.) (MNCN). 2 km al SE de Fuente el Fresno: 1IV-2000, 1 ejemplar (I. Martínez Solano y M. García París leg.) (MNCN). Sierra Madrona: 1 ejemplar (J. Pérez-López leg.) (JLR). Sa . Casalobos: 15-V-1986, 1 ejemplar (JLZ). Solana del Pino, Río Robledillo - Río Frío: 20-IV-1997, 3 ejemplares (M. París y E. Izquierdo leg.) (MNCN). Villar del Pozo: 1-IV-2000, 1 ejemplar (I. Martínez Solano y M. García París leg.) (MNCN). CORDOBA: El Calvario, Cabra: V-1948, 1 ejemplar (Gasull leg.) (MZB). Puente Genil: 13-V-1948, 1 ejemplar (Gasull leg.) (MZB). Sierra de Córdoba, Las Ermitas: 9-V-48, 1 ejemplar (Gasull leg.) (MZB). CUENCA: Camino de las Torcas: 18-III-1991, 1 ejemplar (J. L. Zapata leg.) (JLZ). Nacimiento del Río Cuervo: 7-V-1983, 1 ejemplar (M. Ortego leg.) (AZ). $4 \mathrm{~km}$ al SE de Arcos de la Sierra: 18-V-1998, 2 ejemplares (M. García París y C. Martín leg.) (MNCN). 2 km al E de Arcos de la Sierra: 18-V-1998, 1 ejemplar (M. García París y C. Martín leg.) (MNCN). La Ceja, $10 \mathrm{~km}$ al O de Las Majadas: 11-VII-1998, 1 ejemplar (M. García París y C. Martín leg.) (MNCN). $3 \mathrm{~km}$ al S de Portilla: 18-V-1998, 1 ejemplar (M. García París y C. Martín leg.) (MNCN). 2 km al E de Ribatajada: 18-V-1998, 1 ejemplar (M. García París y C. Martín leg.) (MNCN). $2 \mathrm{~km}$ al NO de Ribatajadilla: 18-V1998, 1 ejemplar (M. García París y C. Martín leg.) (MNCN). Tribaldos: 14-VI-2000, 1 ejemplar (M. García París leg.) (MNCN). GIRONA: Figueras: VIII-1915, 1 ejemplar (P. Benód leg.) (MZB). GRANADA: Benamaurel: 16-IV-1991, 5 ejemplares (J. L. Ruiz leg.) (JLR). Cañada Periate, Iznalloz: 800 m, 26-V-1991, 1 ejemplar (J. A. Hernández leg.) (JLR). Illora: 22-IV-1988, 1 ejemplar (P. Barranco leg.) (JLR). Las Gabias: 29-III-1987, 2 ejemplares (F. Román leg.) (JLR). Peñones de San Francisco, Sierra Nevada, 2400 m: 10-VI1990, 1 ejemplar (P. Barranco leg.) (JLR); 25-VI-1992, 2 ejemplares (J. L. Ruiz leg.) (JLR). Siete Lagunas, Sierra Nevada: 22-VII-1991, 1 ejemplar (J. L. Ruiz leg.) (JLR). GUADALAJARA: Aº de las Fraguas: 18-IV-1998, 1 ejemplar (J. L. Zapata leg.) (JLZ). Torremocha del Campo: 15-V-2000, 1 ejemplar (G. Astudillo leg.) (MNCN). HUELVA: Villablanca: 25-III-1983, 3 ejemplares (J. de Ferrer leg.) (JF y JLR). HUESCA: Mte. Atarés: 27-VII-1963, 1 ejemplar (F. Español leg.) (MZB). Pertusa: VI-1936, 1 ejemplar (Museu) (MZB). Saluenza, Barbastro: 16-V-1933, 1 ejemplar (MZB). JAÉN: Baeza: VI-1923, 1 ejemplar (Codina leg.) (MZB). LLEIDA: Almacellas: V-1923, 4 ejemplares (Novellas leg.) (MZB). MADRID: Madrid: $2 \mathrm{~km}$ al SE de Arganda: 10-VI1998, 1 ejemplar (M. García París y C. Martín leg.) (MNCN). $4 \mathrm{~km}$ al SE de Arganda: 12-V-1998, 1 ejemplar (M. García París y C. Martín leg.) (MNCN). $3 \mathrm{~km}$ al S de Arganda: 5-VI1998, 1 ejemplar (M. García París leg.) (MNCN). El Berrueco: 12-VI-1999, 1 ejemplar (M. García París leg.) (MNCN). 3 km al NO de Camporreal: 16-V-1998, 2 ejemplares (M. García París y C. Martín leg.) (MNCN). Camporreal - Loeches: 26-V2000, 2 ejemplares (M. García París leg.) (MNCN). 5 km al NW de Carabaña: 15-V-2000, 1 ejemplar (M. García París leg.) (MNCN). Casa de la Aldehuela, $5 \mathrm{~km}$ al SO de Torrelaguna: 13-VI-1998, 1 ejemplar (M. García París leg.) (MNCN). Madrid: C. Almodóvar: 14-IV-1989, 1 ejemplar (M.A.S. Sobrino leg.) (MAS); Cerro Almodóvar, Santa Eugenia: 20-V1998, 1 ejemplar (M. García París leg.) (MNCN). Madrid: Pilar Saleta [Hortaleza] (MNCN). Madrid: La Dehesa [de la Villa]: 27-III-1988, 1 ejemplar (JLZ). D. [Dehesa de la] Villa: 20-IV-1980, 1 ejemplar (M.A.S. Sobrino leg.). (MAS). Matalpino [Mataelpino]: 20-III-1995, 1 ejemplar (J. L. Zapata leg.) (JLZ). Hayedo de Montejo - Puerto del Cardoso: 12-VI1999, 1 ejemplar (M. García París leg.) (MNCN). 4 km al S de Pozuelo del Rey, 800 m: 1-V-2000, 1 ejemplar (M. García París leg.) (MNCN). $1 \mathrm{~km}$ al SO de Valdaracete, $800 \mathrm{~m}: 1-\mathrm{V}-2000,1$ ejemplar (M. García París leg.) (MNCN). $3 \mathrm{~km}$ al E de Valdaracete: 15-V-2000, 1 ejemplar (M. García París leg.) (MNCN). $3 \mathrm{~km}$ al S de Valdaracete, $790 \mathrm{~m}: 25-\mathrm{III}-2000,1$ ejemplar (M. García París leg.) (MNCN). $2 \mathrm{~km}$ al E de Valdilecha: 15-V-2000, 1 ejemplar (M. García París leg.) (MNCN). $3 \mathrm{~km}$ al $\mathrm{O}$ de Villamanrique de Tajo: $580 \mathrm{~m}$, VK7736: 24-III-2000, 1 ejemplar (M. García París leg.) (MNCN). $4 \mathrm{~km}$ al N de Villarejo de Salvanés: 15-V-2000, 1 ejemplar (M. García París leg.) (MNCN). MÁLAGA: El Quejigar, Sierra de las Nieves: 25-IV-1991, 5 ejemplares (J. L. Ruiz leg.) (JLR). Fuentepiedra: IV-1989, 1 ejemplar (J. L. Ruiz leg.) (JLR). MURCIA: Roche, Cartagena: 15-IV-1996, 2 ejemplares (J. Negro leg.) (JLR). NAVARRA: Tafalla: VI-1940, 1 ejemplar (Goñi leg.) (MZB); 1940, 1 ejemplar (P. Goñi leg.) (MZB). OURENSE: Castrocaldelas: IV-1965, 1 ejemplar (Rodríguez leg.) (MZB). SALAMANCA: $4 \mathrm{~km}$ al E de Boadilla: 3-IV-1999, 1 ejemplar (M. García París y C. Martín leg.) (MNCN). Cabrerizos: 30-III-1985, 1 ejemplar (J. Angel leg.) (USal); 19-V-1987, 1 ejemplar (Durante leg.) (USal). Castillo del Buen Amor: 18-IV-1985, 1 ejemplar (A. Martín Pérez leg.) (USal). Cilleros: 16-IV-1985, 1 ejemplar (M. Burque leg.) (USal). [Ciudad Rodrigo]: Valhondo: 12-III-1985 (J. Martín Azabal leg.) (USal). ISPE [Salamanca]: 2-X-1983, 1 ejemplar (J. C. V. leg.) (USal). La Flecha: 18-III-1987, 1 ejemplar (Larregola leg.) (USal); V-1987, 1 ejemplar (M. J. Ruano leg.) (USal); V-1987, 1 ejemplar (L. Calabia leg.) (USal); 9-V1987, 1 ejemplar (A. Gil leg.) (USal); 9-V-1987, 1 ejemplar (J. L. G. leg.) (USal); 10-V-1987, 1 ejemplar (B. Mullio leg.) (USal); 10-V-1987, 1 ejemplar (M. A. García leg.) (USal); 12V-1997, 1 ejemplar (A. D. Masaf leg.) (USal); 24-V-1989, 1 ejemplar (L. Mdez. leg.) (USal). La Fregeneda: 4-IV-1987, 1 ejemplar (J. Barajas leg.) (USal); 2-V-1987, 1 ejemplar (P. García Gómez leg.). Navasfrías: 18-II-1987, 1 ejemplar (M. Calzada leg.) (USal); 18-V-1987, 1 ejemplar (A. L. Fernández leg.) (USal). Pto. Perales: 18-V-1984, 1 ejemplar (J. Maez leg.) (USal); 18-V-1987, 1 ejemplar (M. P. García leg.) (USal); 19V-1987, 1 ejemplar (A. Redondo leg.) (USal). Puente Salud: 10-V-1986, 1 ejemplar (P. Martín leg.) (USal). Retortillo: 1 ejemplar (MNCN); 20-IV-1985, 1 ejemplar (P. Domingo leg.) (USal). Robleda: 19-IV-1982, 1 ejemplar (J. A. Pascual leg.) (USal). Salamanca: 27-IV-1985, 1 ejemplar (A. Romano leg.) (USal); 20-VI-1985, 1 ejemplar (I. Sarasola leg.) (USal); 15-V1986, 1 ejemplar (A. Andaces leg.) (USal); 19-V-1986, 1 ejemplar (G. leg.) (USal). Tamames: 11-IV-1985, 1 ejemplar (J. A. Rodríguez leg.) (USal). $3 \mathrm{~km}$ al E de Vilvestre: 2-IV-1999, 1 
ejemplar (M. García París y C. Martín leg.) (MNCN). SEGOVIA: Cañicosa: 4-VII-1999, 5 ejemplares (M. García París y C. Martín leg.) (MNCN). La Granja: V-1906, 2 ejemplares (MZB). SEVILLA: Marchena: 23-III-1986, 2 ejemplares (O. Escolá leg.) (MZB). SORIA: Castillo de Medinaceli: 7-VI1997, 1 ejemplar (J. París y M. García Rollán leg.) (MNCN) Pto. Oncala: VII-1965, 1 ejemplar (Esp. Negre leg.) (MZB); 23-V-1998, 1 ejemplar (P. Bahillo leg.) (MNCN). Soria: 14-IV1987, 1 ejemplar (J. D. Molinar leg.) (USal). TARRAGONA: Capsanes: 21-III-1981, 2ex. (J. y E. Vives leg.) (AZ). Camí de Prades, Espluga de F.: 23-V-1919, 1 ejemplar (Codina leg.) (MZB). Espluga de Francoli: 19-V-1919, 1 ejemplar (Codina leg.) (MZB). Falset: 1 ejemplar (Museu leg.) (MZB). Valls: 1 ejemplar (Col. Español) (MZB). TERUEL: Frías, Albarracín, VII-1958, 4 ejemplar. (F. Español leg.) (MZB). 4 km al SO de Peñarroya de Tastavins, 19-VI-1998, 1 ejemplar (M. García París y C. Martín leg.) (MNCN). TOLEDO: Puerto del Milagro: 1-IV-1988, 1 ejemplar (JLZ). Quero: 24-III-1990, 1 ejemplar (JLZ). Quero: 1 ejemplar (A. Montes leg.) (MNCN). Risco de las Paradas, Navahermosa: 2-VI-1966, 1 ejemplar (Esp. Neg. leg.) (MZB). ZAMORA: Brime de Urz: 13-IV1992, 1 ejemplar (P. Bahillo leg.) (MNCN); 29-VI-1992, 2 ejemplares (P. Bahillo leg.) (MNCN). Cubo de Benavente: 18IV-1987, 1 ejemplar (A. J. Cuevas leg.) (USal). Granucillo de Vidriales, TM5759: 17-IV-1992, 3 ejemplares (P. Bahillo leg.) (MNCN); 26-IV-1992, 1 ejemplar (P. Bahillo leg.) (MNCN). Morales del Rey: 1-V-1994, 1 ejemplar (P. Bahillo leg.) (MNCN). Santibáñez de Vidriales: 18-IV-1992, 1 ejemplar (P. Bahillo leg.) (MNCN). ZARAGOZA: Bujaraloz: 15-IV-1968, 1 ejemplar (F. Español leg.) (MZB). PORTUGAL: ALGARVE: Barragem de Bravura: 15-V-1982, 1 ejemplar (A. Zuzarte leg.) (AZ). Foia, Serra de Monchique: 20-IV-1982, 4 ejemplares (T. Branco leg.) (AZ). Serra de Monchique: 25-IV-1978, 1 ejemplar (A. Zuzarte leg.) (AZ). ALTO ALENTEJO: ElvasCaia: 21-III-1989, 1 ejemplar (A. Zuzarte leg.) (AZ). Evora: 19-IV-1979, 1 ejemplar (A. Carela leg.) (UEv). Evora: Estrada de Montemor a $8 \mathrm{~km}$ de Evora: 22-V-1980, 1 ejemplar (A Zuzarte y E. Luna leg.) (AZ). Mitra: 20-IV-1979, 1 ejemplar (Raimundo leg.) (UEv.). S. Cristovão, $\mathrm{EN} \mathrm{n}^{\circ}$ 253, Km 57: 9-IV1982, 6 ejemplares (A. Zuzarte leg.) (AZ). St. Amaro-Sousel: 21-IV-1968, 1 ejemplar (A. Zuzarte leg.) (AZ); 2-IV-1977, 2 ejemplares (A. Zuzarte leg.) (AZ). Val das Quintas-Veiros: 31III-1968, 1 ejemplar (A. Zuzarte leg.) (AZ); 3-IV-1986, 1 ejemplar (A. Zuzarte leg.) (AZ). BAIXO ALENTEJO: Beja, Neves: 3-V-1983, 1 ejemplar (A. Zuzarte leg.) (AZ). Ervidel: 3-V1983, 1 ejemplar (A. Zuzarte leg.) (AZ). Vilaverde de Ficalho a Do Pinto: 9-IV-1983, 3 ejemplares (T. Branco leg.) (AZ). BEIRA ALTA: Sabugal: 6-VII-1981, 1 ejemplar (A. Zuzarte leg.) (AZ). RIBATEJO: Coruche-Bairro do Serração: 26-III1977, 2 ejemplares (A. Zuzarte leg.) (AZ). Foros de Benfica: 30-V-1982, 4 ejemplares (A. Zuzarte leg.) (AZ). Muge: 13-V1967, 5 ejemplares (T. Branco leg.) (AZ). TRAS-OS-MONTES: Bragança-Rebordainhos: 11-VI-1983, 2 ejemplares (A. Zuzarte leg.) (AZ). Serra de Bornes: 26-IV-1984, 2 ejemplares (T. Branco leg.) (AZ).

La cita del "Puerto de Lorre" (Bologna, 1989) posiblemente corresponda al Puerto de Torre Miró (Castellón). En la colección del MZB existe un ejemplar con etiqueta: Eivissa, V19, (Font Quer leg.) (MZB) que posiblemente corresponda a una introducción accidental o a un error de etiquetado.

Los ejemplares examinados y citas bibliográficas corresponden a las siguientes provincias y cuadrículas UTM de 10x10 km: España: Albacete (WH48, WH51, WH56, WH67, WJ42, WJ91, XJ10); Alicante (XH86, XH87, XH96, YH01, YH03, YH04, YH07, YH14, YH15, YH17, YH25, YH27, YH39, YH48, YH49); Almería (WF49, WF68, WF77, WG50, WG52, WG67, WG71, WG80); Avila (UK15, UK25, UK46, UK79, UK88, UL50); Badajoz (PD70, QC03, QC33, TJ60); Barcelona (DF17, DF18, DF28, DF29, DF38, DG12, DG20, DG22, DG23, DG32, DG33, DG34, DG40, DG52, DG70); Burgos (UM88, VM35, VM38, VM41, VM48, VM58, VM64, VM83, VN20); Cáceres (QD36, QE04, QE43, QE45, TJ52, TJ58, TJ74, TJ96, TK36, TK44, TK56, UJ17); Cádiz (QA53, QA54, QA56, QA65, TE68, TF53, TF70, TF80, TF81); Castellón (YK28, YL40, YL50); Ciudad Real (UH85, UH98, UJ36, UJ61, UJ90, VH05, VJ00, VJ02, VJ10, VJ11, VJ13, VJ20, VJ30, VJ34, VJ72, VJ86); Córdoba (TH95, UG08, UG39, UG44, UG49, UG74, UH40); Cuenca (WK02, WK73, WK75, WK76, WK78, WK81, WK83, WK96, XK06); Girona (DG97); Granada (VG22, VG32, VG40, VG41, VG53, VG54, VG60, VG61, VG70, VG83, VG90, VG92, WG24, WG25, WG26, WG35, WH30, WH50); Guadalajara (VK88, VL73, VL83, WL33); Huelva (PB43, PB66, QB34, QC30); Huesca (BG55, BG61, BG65, XN82, YM35, YN01); Jaén (VG27, VH01, VH41, VH54, VH50, VH65, WG09); La Rioja (VM96, WM35, WN40); León (PH81, QG37, QG38, QG39, QG47, QH01, QH10, QH15, QH20, QH22, QH40, QH42, TM68, TM79, TM86, TM98, TN61, TN62, TN64, TN65, TN71, TN73, TN74, TN81, TN82, TN84, TN85, TN90, TN91, TN92, TN93, TN94, TN95, UM39, UN03, UN04, UN11, UN14, UN16, UN23, UN24, UN30, UN32, UN33); Lleida (BG82); Madrid (VK36, VK37, VK38, VK46, VK47, VK48, VK56, VK57, VK58, VK59, VK64, VK65, VK66, VK67, VK73, VK74, VK75, VK76, VK85, VK86, VL00, VL10, VL11, VL20, VL30, VL50, VL51, VL52, VL54); Málaga (UF16, UF76, UG41, UG72); Murcia (XG86, XH46); Navarra (XN00); Ourense (PG39); Salamanca (PE86, PE95, PF74, PF95, QE07, QE19, QE49, QF22, QF31, QF40, TK67, TL73, TL75, TL83, TL85); Segovia (UL90, VL12, VL35); Sevilla (QB47, TG34, TG43, TG65, TG83, TG86, TH50); Soria (WL45, WM42, WM54); Tarragona (CF15, CF48, CF57); Teruel (BF41, XK07, XK16, XK18, XK26, XK27, XK37, XK55, XK66, XK84, XK87, YK07, YK17, YL16, YL44); Toledo (UK72, UJ78, UJ97, VJ77, VK11); Valencia (YH09, YJ08, YJ27); Valladolid (UL17); Vizcaya (regional); Zamora (QG26, QG46, TL87, TL97, TM55, TM65, TM66); Zaragoza (WM92, XL15, XL47, XL69, XM03, XM61, XM70, XM71, XM82, XM84, XM90, YL09, YL27, YL39, YL46, YM12, YM31). Gibraltar (TF80). Portugal: Algarve (NB20, NB22, NB32, NB53); Alto Alentejo (NC56, NC86, NC96, NC97, PD11, PD21, PD60, NC56, NC86); Baixo Alentejo (NC80, NC90, PC40); Beira Alta (PE66); Beira Litoral (NE45); Estremadura (MC68, MC88); Ribatejo (ND22, ND23, ND41); Tras-os-Montes (PF68; PG83) (Fig. 2).

\section{Lagorina Mulsant et Rey, 1858}

El género Lagorina, bien caracterizado por rasgos de las antenas y por el color del tegumento verde metálico, incluye dos especies de la región mediterránea occidental (Pardo Alcalde, 1950; 


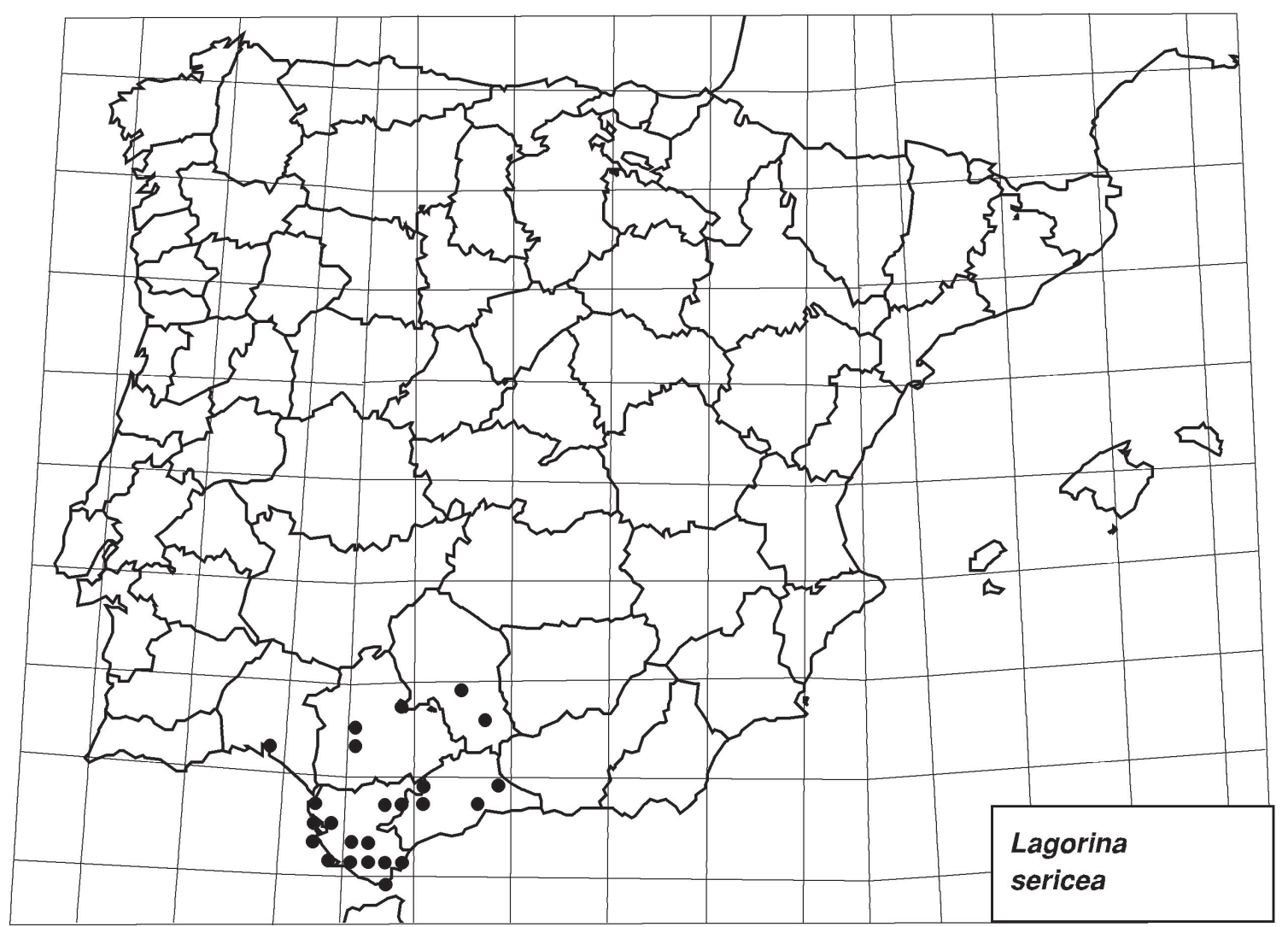

Fig. 3.- Mapa de la Península Ibérica con los puntos correspondientes a las localidades donde se ha localizado o citado en la bibliografía Lagorina sericea (Waltl, 1835). Cada punto equivale a una cuadrícula UTM de 20 x $20 \mathrm{~km}$.

Fig. 3.- Map of the Iberian Peninsula with locality records for Lagorina sericea (Waltl, 1835). Each dot represents a $20 \times 20 \mathrm{~km}$ UTM square.

Bologna, 1991), una de ellas, L. sericea, se encuentra en el sur peninsular.

\section{Lagorina sericea (Waltl, 1835)}

Esta especie se localiza en el extremo noroccidental de África y en el extremo meridional de la Península Ibérica (Borchmann, 1917; Mader, 1927; Pardo Alcaide, 1950). Górriz Muñoz (1882) y Rodríguez López-Neyra (1914) señalan su presencia en Andalucía. Se ha citado en la provincia de Cádiz: Algeciras (Rosenhauer, 1856; Sumakov, 1934) y Chiclana (Medina, 1895); en la de Málaga: Málaga (Cobos, 1949; Torres Sala, 1962); en la de Sevilla: Sevilla (Medina, 1895), y en Gibraltar (Champion, 1891). Fuente (1933) señala las mismas provincias.

Las poblaciones ibéricas de la especie se muestran muy homogéneas, con escasa variabilidad en la talla y en la coloración. Las únicas variaciones de coloración observadas corresponden a la extensión de los reflejos rojizos o azulados sobre el fondo verde metálico típico. Los adultos se muestran activos entre finales de marzo y mediados de mayo. Según observaciones efectuadas en la provincia de Cádiz (J. de Ferrer, com. pers.; datos propios) los imagos se han encontrado sobre Ranunculaceae de flores amarillas.

MATERIAL EXAMINADO: Se han examinado 308 ejemplares de las localidades siguientes: ESPAÑA: CADIZ: Albergue Juvenil, El Bosque: 2-IV-1992, 1 ejemplar (M. Baena leg.) (MB). Alcalá de los Gazules: 8-IV-1986, 1 ejemplar (M. García-París leg.) (MNCN). Algeciras: 21-IV-1921, 3 ejemplares (A. Codina leg.) (MZB); 22-IV-1921, 30 ejemplares (Novellas leg.) (MZB); 23IV-1921, 66 ejemplares (A. Codina leg.) (MZB); 24-IV-1921, 1 ejemplar (A. Codina leg.) (MZB); 25-IV-1921, 4 ejemplares (Novellas leg.) (MZB); 26-IV-1921, 2 ejemplares (A. Codina leg.) (MZB); 1 ejemplar (MNCN); VI-1901, 3 ejemplares (Escalera leg.) (MNCN); 44 ejemplares (C. Bolívar leg.) (MNCN). Benalup de Sidonia (Casas Viejas): 7,8-IV-1914, 43 
ejemplares (C. Bolívar leg.) (MNCN). Carretera CampillosOlvera, Olvera: 20-III-1986, 1 ejemplar (M. Baena leg.) (MB). Chiclana: 31-IV-1922, 1 ejemplar (Col. Español) (MZB). Chiclana: 1914, 4 ejemplares (Fallen leg.) (MNCN). Conil: 1925 (Ceballos leg.) (MNCN). El Cerro, San Fernando: 21-III-1992, 2 ejemplares (P. Coello leg.) (JLR). El Palmar, Véjer de la Frontera: 26-III-2002, 1 ejemplar (J. L. Zapata leg.) (JLZ). Getares, Algeciras: 5-V-1974, 1 ejemplar (J. de Ferrer leg.) (JF); 5-IV-1982, 1 ejemplar (JF); 10-V-1987, 2 ejemplares (JLR). Jerez: 1 ejemplar (E. Pons leg.) (MNCN). Jerez Front[era]: IV1935, 1 ejemplar (Benítez, leg.) (MZB). La Janda: V-1976, 2 ejemplares (Ramírez leg.) (MZB). Laguna de Janda: 1 ejemplar (W. Marten leg.) (MZB). La Nava, Chiclana de la Frontera: 10V-1993, 2 ejemplares (P. Coello leg.) (JLR); 26-III-1995, 3 ejemplares (JLR). Laguna de Medina, Jerez: 27-III-1983, 2 ejemplares (J. de Ferrer leg.) (JF). Los Barrios: 26-IV-1991, 4 ejemplares (J. L. Ruiz leg.) (JLR). Medina [Sidonia]: 1 ejemplar (E. Barón leg.) (AZ). Ptno. Hurones, Jerez: 5-IV-1977, 2 ejemplares (J. de Ferrer leg.) (JF). Puerto de Sta. María: 6 ejemplares (E. Marvier leg.) (MZB). San Fernando: 21-III-1986, 1 ejemplar (P. Coello leg.) (JLR). San Roque: V-1979. 4 ejemplares (J. Ramírez leg.) (AZ). Tarifa: 1 ejemplar (MNCN); V-1903, 28 ejemplares (Escalera leg.) (MNCN). Ubrique: 19-IV-1980, 4 ejemplares (J. de Ferrer leg.) (JF y JLR). CORDOBA: Córdoba: 3 ejemplares (Amor leg.) (MNCN). La Nava, Cabra: 30-V-1992, 1 ejemplar (JLR). HUELVA: La Ribera: 25-III-2000, 3 ejemplares (M. Huertas leg.) (SAE); 21-IV-2000, 8 ejemplares (SAE). MALAGA: Facultad de Ciencias, Málaga: 13-IV-1987, 1 ejemplar (A. Ruiz leg.) (JLR). Málaga: 1 ejemplar (Aragoncillo leg.) (MNCN), 1 ejemplar (Lozano leg.) (MNCN). Sierra de las Nieves, Ronda: 29-V-1982, 1 ejemplar (J. de Ferrer leg.) (JF). Villanueva del Rosario (UF8094): 14-V-1988, 1 ejemplar (Alonso-Zarazaga leg.) (MNCN). SEVILLA: Campana: 1 ejemplar (Calderón leg.) (MNCN). La Salud: III-1941, 2 ejemplares (A. Benitez leg.) (MNCN); IV-1941, 4 ejemplares (A. Benitez leg.) (MNCN). Sevilla: 5 ejemplares (Dusmet leg.) (MNCN).

Los ejemplares examinados y citas bibliográficas corresponden a las siguientes provincias y cuadrículas UTM de 10x10 km: España: Cádiz (QA43, QA45, QA53, QA56, QA61, QA65, TE68, TE79, TF31, TF33, TF41, TF42, TF53, TF70, TF76, TF77, TF80, TF86, UF09); Córdoba (UG49, UG75); Huelva (PB82); Málaga (UF16, UF66, UF76, UF89); Sevilla (TG33, TG34, TG86). Gibraltar (TF80).

\section{Lytta Fabricius, 1775}

Género de amplia distribución holártica con penetraciones en el neotrópico y en Asia tropical que comprende cerca de 120 especies distribuidas en 8 subgéneros (Bologna, 1991). De éstas, únicamente $L$. vesicatoria, del subgénero nominal, se encuentra en Europa Occidental.

\section{Lytta (Lytta) vesicatoria (Linnaeus, 1758)}

Especie extendida por la mayor parte de Europa y Asia paleártica, de la que se reconocen en la actualidad cuatro subespecies; la nominal, presente en la Península Ibérica (Bologna, 1991). A pesar de que ha sido repetidamente citada de todo el territorio peninsular (Rodríguez López-Neyra, 1914; Valladares y Salgado, 1983) e incluso se ha señalado como muy abundante en Andalucía (Cuní Martorell \& Martorell Peña, 1876; Górriz Muñoz, 1882), nosotros no hemos visto ningún ejemplar capturado en el sur de la Península, por lo que consideramos que esas citas posiblemente correspondan a confusiones con Lagorina sericea. De acuerdo con los ejemplares examinados el límite meridional de distribución de $L$. vesicatoria se localiza a lo largo del Sistema Central con penetraciones hasta la Serra de São Mamede en Portugal (Fig. 4).

Los ejemplares ibéricos presentan una variabilidad muy alta en la talla que no parece guardar relación con la procedencia geográfica de los ejemplares. Todos los individuos examinados muestran una coloración general verde metálica con reflejos cobrizos más o menos extendidos, excepto diez ejemplares de Madrigal (J. Ardois leg.) que son de un color bronce oscuro y un ejemplar de Tiermas (A. Sanz leg.) que es de color azul oscuro metálico.

La fenología de los adultos se extiende de abril a agosto como ya ha sido señalado por otros autores (Bologna, 1991; Valladares y Salgado, 1983). Como señala Valladares Díez (1984), a diferencia del resto de las poblaciones europeas, todos los ejemplares ibéricos con datos precisos de captura se encontraron sobre flores de escobas y piornos del género Cytisus (J. A. Zuzarte y F. A. Montes com pers. y datos propios), y únicamente Cuní Martorell (1881) señala su captura en los Pirineos catalanes sobre fresnos (Fraxinus).

Cuní Martorell \& Martorell Peña (1876) y Cuní Martorell $(1881,1888)$ citan la especie de los alrededores de Puigcerdá (Gerona) y de las orillas del Besós y Llobregat (Barcelona). Górriz Muñoz (1882) recoge las citas anteriores de Cataluña, la de Graells de Madrid, la de Amor de Córdoba, y añade Madrid y Escorial (Madrid), Lerma (Burgos), Zaragoza y Cariñena (Zaragoza) y Milagro (Navarra). Oliveira (1894) cita la especie de Bragança, Cedaes, Gerez, Caramulo, Coimbra, Guarda y Penamacôr. Rivas Mateos (1897) señala la presencia de la especie a $2420 \mathrm{~m}$ de altitud en los Riscos del Tejadillo, Sierra de Béjar (Salamanca). Górriz Muñoz (1902) repite la cita de Cariñena. Champion (1903) observa la especie en Piedralaves (Avila) y Béjar (Salamanca). Navás (1904) la cita en el Moncayo. Rodríguez López-Neyra (1914) indica la presencia de la especie en "Toda España" sin aportar nuevas localidades. Iglesias (1928) cita 


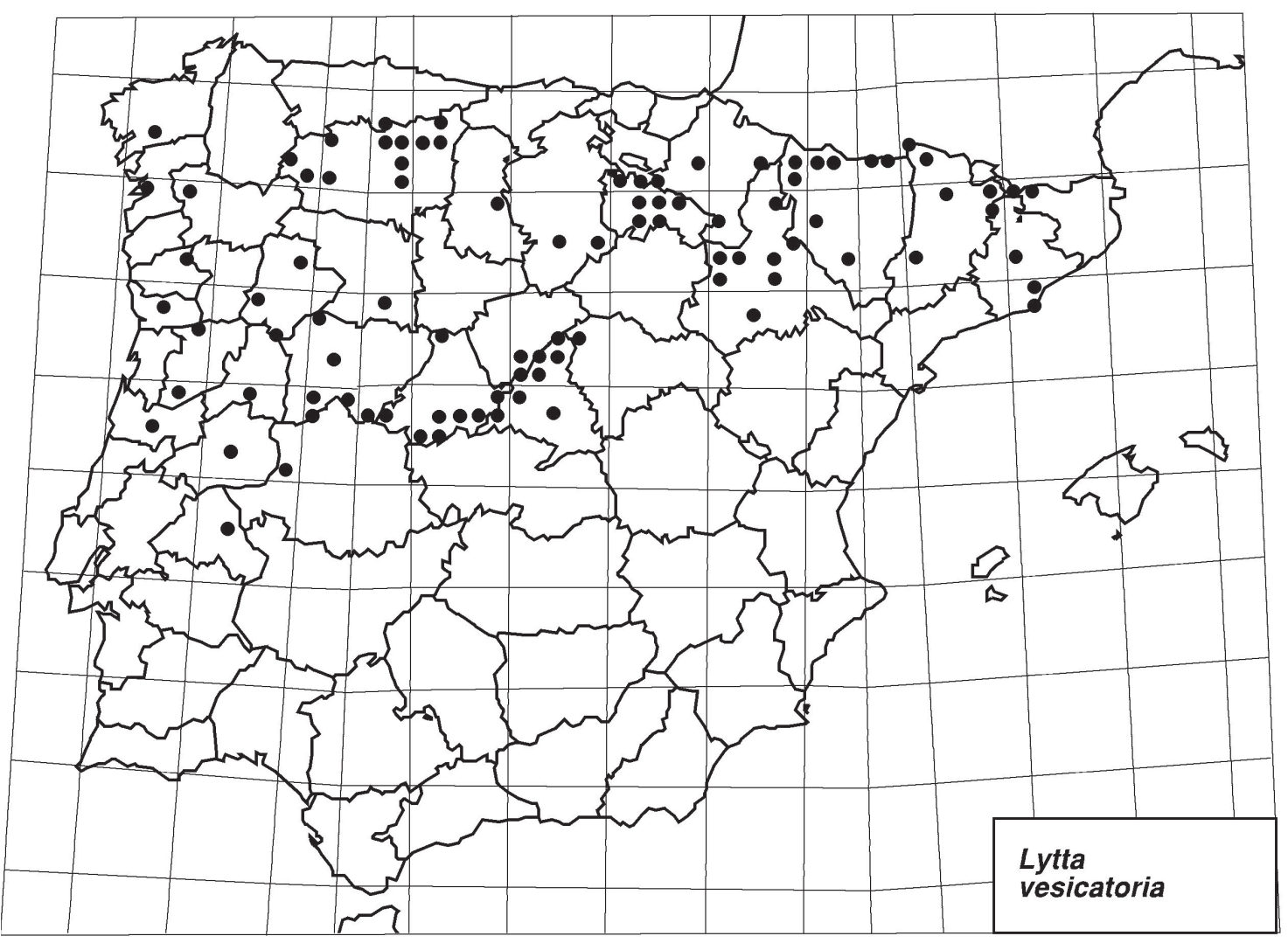

Fig. 4.- Mapa de la Península Ibérica con los puntos correspondientes a las localidades donde se han localizado o citado en la bibliografía ejemplares de Lytta (Lytta) vesicatoria (Linnaeus, 1758). Cada punto equivale a una cuadrícula UTM de 20 x $20 \mathrm{~km}$.

Fig. 4. - Map of the Iberian Peninsula with locality records for Lytta (Lytta) vesicatoria (Linnaeus, 1758). Each dot represents a 20 x $20 \mathrm{~km}$ UTM square.

la especie en Santiago (A Coruña). Valladares \& Salgado (1983) y Valladares Díez (1984) aportan numerosas citas de León; Serrano (1983) la cita en Vila Real. Pérez Moreno \& Cabrero Sañudo (1999) en La Rioja y Recalde et al. (2002) en Aragón recogen los datos conocidos, incluida una cita de Asso (1784) en Jarque (Zaragoza), y aportan numerosas citas nuevas.

MATERIAL EXAMINADO: Se han examinado 323 ejemplares de: ESPAÑA: Extremadura: 1 ejemplar (MNCN). ASTURIAS: Puerto de Pajares: 2 ejemplares (A. Kricheldorff leg.) (MNCN). AVILA: El Tiemblo: 10-V-1980, 2 ejemplares (J. I. López-Colón leg.) (JF). Madrigal [de las Altas Torres]: 13 ejemplares (J. Ardois leg.) (MNCN). Ramacastañas: 12-IV1985, 2 ejemplares (M. De los Mozos leg.) (JLR). Sierra de Gredos: 22 ejemplares (MNCN). Valle de Iruelas: V-1920, 1 ejemplar (C. Bolívar leg.) (MNCN). Villarejo del Valle: 1 ejemplar (MNCN); 2 ejemplares (Lauffer leg.) (MNCN). BARCELONA: Artès: 12-VI-1927, 1 ejemplar (A. Villarrubia leg.) (MZB). BURGOS: Moncalvillo: 1 ejemplar (MNCN). CÁCERES: Hervás: 18-V-1976, 1 ejemplar (G. Muñoz leg.)
(USal). Zarza la Mayor: 2-IV-1985, 1 ejemplar m (B. Antunez leg.) (USal); 29-VIII-1985, 1 ejemplar (B. Antunez leg.). GIRONA: La Molina (Pyr. Or.): VII-1953, 1 ejemplar (Schutte leg.) (MZB). Nas (Cerdanya), $1350 \mathrm{~m}$ : 3-VIII-76, 1 ejemplar (L. Domenech leg.) (MZB). Rivas [Ribes de Freser]: 1 ejemplar (Zariquiey leg.) (MZB). Vall de Ribas: 1928, 1 ejemplar (A. Villarrubia leg.) (MZB). GUADALAJARA: Portillo del Lobo: 13-VI-1918, 3 ejemplares (Expedición del Instituto Español de Entomología) (MNCN). HUESCA: Panticosa: VIII-1942, 1 ejemplar (Montada leg.) (MZB); VIII-1943, 1 ejemplar (Montada leg.) (MZB). Balneario de Panticosa: 1 ejemplar (C. Bolívar leg.) (MNCN); Bujaruelo: 1338 m, 1 ejemplar (Expedición del Instituto Español de Entomología) (MNCN). Embún: V-1952, 1 ejemplar (L. Báguena leg.) (MNCN). Panticosa: 1 ejemplar (Dusmet, leg.) (MNCN). Sallent de Gállego: 25-VI-1966, 3 ejemplares (AZ). LA RIOJA: Cameros: 1 ejemplar (C. Bolívar) (MNCN). Navarrete: 11 ejemplares (MNCN). San Román de Cameros: VII-1940, 1 ejemplar (Parra leg.) (MNCN). LEON: Cistierra [Cistierna]: 11-VI-1945, 1 ejemplar (W. Marten leg.) (MZB). Reserva de Caza de Mampodre: 24-VI-1986, 1 ejemplar (M. García-París leg.) (MNCN). Riaño: VI-1946, 1 ejemplar (Marten leg.) 
(MZB). Valdeteja, $1100 \mathrm{~m}$ : 10-VI-1983, 1 ejemplar (J. M. Ávila leg.) (JLR). LLEIDA: [Torre de] Capdella: VII-1923, 1 ejemplar (Novellas leg.) (MZB). San Pere Forcats, Vall d'Aran, Estanys Colomers: VII-1934, 5 ejemplares Museu leg.) (MZB) Tuixent: 22-VII-1920, 5 ejemplares (Codina leg.) (MZB); Tuixent, Rivera del R. Josa: 22-VII-1920, 4 ejemplares (Codina leg.) (MZB). Vallfogona de Balaguer: 1 ejemplar S. Maluquer leg.) (MZB). Vall de Joeu, Vall d'Aran: 9-VII-1990, 1 ejemplar (X. Vázquez leg.) (JLR). MADRID: El Berrueco: 12-VI-1999, 1 ejemplar (M. García París leg.) (MNCN). Cercedilla: 2 ejemplares; 1 ejemplar (J. Ardois leg.) (MNCN); 40 ejemplares (C. Bolívar leg.) (MNCN); 1 ejemplar (J. Lauffer leg.) (MNCN); 3 ejemplares (García Mercet leg.) (MNCN); 1 ejemplar (J. Abajo leg.) (MNCN); 1 ejemplar (Moróder leg.) (MNCN); 4 ejemplares (Expedición del Museo) (MNCN); VI-1927, 6 ejemplares (MNCN); V-1961, 1 ejemplar (Messerschmidt leg.) (MNCN). Cercedilla: El Ventorrillo, 1480 m, VI-1965, 1 ejemplar (MNCN). Cercedilla: Estación Alpina, 1500 m, VIII-1935, 1 ejemplar (J. Hernández leg.) (MNCN). Cercedilla: La Peñota, 19-IV-1945, 1 ejemplar (MNCN). $5 \mathrm{~km}$ al N de Cercedilla: 16VI-2000, 7 ejemplares (M. Moreli leg.) (MNCN). El Escorial: 17 ejemplares (MNCN); 3 ejemplares (C. Bolívar leg.) (MNCN); 1 ejemplar (G. Carrasco leg.) (MNCN); 20-VI-1905, 1 ejemplar (G. Mercet leg.) (MNCN); VI-1926, 1 ejemplar (MNCN). El Escorial: Fuente de la Teja, 2 ejemplares (MNCN). El Escorial: Urbanización Los Arroyos, 25-V-1985, 2 ejemplares (C. Rey leg.) (MNCN). Madrid: 1 ejemplar (MNCN); 1 ejemplar (A. Sanz leg.) (MNCN). La Pedriza: 28V-1989, 2 ejemplares (JLZ). Montejo de la Sierra: 6 ejemplares (C. Bolívar leg.) (MNCN). La Barranca (Navacerrada), 5VI-2000, 2 ejemplares (M. García París leg.) (MNCN) Navalquejemplarigo: 17-V-1957, 9 ejemplares (J. Alvarez leg.) (MNCN). Puerto de Somosierra: 6-VI-1999, 5 ejemplares (M García París y C. Martín leg.) (MNCN). Robledo de Chavela: 1 ejemplar (Expedición del Museo) (MNCN). 4 km S Robledo de Chavela, base Pico Almenara: 10-V-1998, 5 ejemplares (M. García París y C. Martín leg.) (MNCN). Rascafría: Camino Laguna de los Pájaros: 20-VI-2000, 1 ejemplar (M. García París leg.) (MNCN). Rozas de Puerto Real: 1 ejemplar (MNCN). Somosierra: 14-VI, 4 ejemplares (MNCN). Valdemanco: 25-V-2000, 5 ejemplares (M. García París leg.); V-1999, 1 ejemplar (M. García París leg.) (MNCN). Villalba: 1 ejemplar (MNCN); 2 ejemplares (J. Ardois leg.) (MNCN); 9VI-1984, 2 ejemplares (C. Rey leg.) (MNCN). NAVARRA: Estella: 2 ejemplares (MNCN); 2 ejemplares (L. Navarro leg.) (MNCN). OURENSE: Carballino: 2 ejemplares (G. Varela, leg.) (MNCN). PALENCIA: Lantadilla: 25-VIII-1984, 1 ejemplar (T. García leg.) (USal). PONTEVEDRA: Lourizán: V1949, 1 ejemplar (Rodríguez leg.) (MNCN). SALAMANCA: Buenamadre: VI-1959, 1 ejemplar (Alonso leg.) (MZB). Cepeda (Sanz leg.) (MNCN); Candelario: 1800 m, 1 ejemplar (Expedición del Instituto Español de Entomología) (MNCN). Dehesa Candelario: 7-VII-1999, 1 ejemplar (J. R. Verdú leg.) (MNCN). Ciudad Rodrigo: 25-V-1987, 1 ejemplar (R. M. leg.) (USal). La Fregeneda: 15-V-1987, 1 ejemplar (A. Calabuig leg.) (USal). [La Fregeneda]: Vega Torrón: 12-V-1986, 1 ejemplar (L. Pablos leg.) (USal). Orilla Agueda: 18-V-1987, 1 ejemplar (B. Escuadra leg.) (USal). Sierra de Gata: 18-V-1997, 1 ejemplar (P. García Gómez leg.) (USal). SEGOVIA: La Granja de San Ildefonso: 1 ejemplar (MNCN); 1 ejemplar (J. Ardois leg.) (MNCN); 2 ejemplares (Peris Torres leg.) (MNCN). La Granja: El Robledo, 10-VI-1973, 3 ejemplares (Cañeque
Hernández leg.) (MNCN). Valsaín: 8-VI-1991, 1 ejemplar (JLZ). La Panera [San Rafael]: 18-VI-1979, 1 ejemplar (JLZ). SORIA: Cepeda: 1 ejemplar (MNCN). ZARAGOZA: Borja: 10-VIII-1955, 1 ejemplar (S. V. Peris leg.) (MNCN). Tiermas: 31 ejemplares (A. Sanz leg.) (MNCN). ZAMORA: Arribes Duero: 4-V-1989, 1 ejemplar m (P. García leg.) (USal). Zamora: 12-VI-1983, 1 ejemplar m (J. López leg.) (USal). PORTUGAL: ALTO ALENTEJO: Serra São Mamede, Portalegre: 4-V-1998, 4 ejemplares (A. Zuzarte leg.) (AZ). BEIRA BAIXA: [Castelo Branco]: Enguías: 15-IV-1961, 2 ejemplares (AZ); V-1962, 1 ejemplar (AZ). DOURO LITORAL: Serra do Marão: 16-VI-1974, 9 ejemplares (T. Branco leg.) (AZ). MINHO: Serra de Peneda [Gêrez]: 16-VI-1973, 2 ejemplares (T. Branco leg.) (AZ).

Los ejemplares examinados y citas bibliográficas corresponden a las siguientes provincias y cuadrículas UTM de 10x10 km: España: A Coruña (NH34); Asturias (TN76); Avila (UK05, UK25, UK36, UK56, UK67, UK77, UL35); Barcelona (DF27, DF38, DG12); Burgos (VM35, VM84); Cáceres (PE81, TK56); Girona (CG99, DG18, DG19, DG38); Guadalajara (VL65); Huesca (BH62, BH92, XN82, XN90, YM16, YM42, YN13, YN23, YN33); La Rioja (WN11, WN20, WM27, WM37, WM39, WN40, WM47, WM58, WM59, WM78, WM79); León (PH62, PH72, PH90, QH01, QH14, TN64, TN80, TN81, TN84, TN92, TN94, TN95, UN05, UN24, UN25, UN26, UN35); Lleida (CG12, CG39, CG87, CH13, CH22); Madrid (UK76, UK98, VK09, VK19, VK47, VL01, VL11, VL21, VL22, VL31, VL42, VL52, VL54, VL55); Navarra (WN82, XM07); Ourense (NG79); Palencia (UM88); Pontevedra (NG29); Salamanca (PF74, QE06, QE09, QE19, QE58, QF32, TK66, TK67); Segovia (VL01, VL12); Zaragoza (XL47, XM03, XM10, XM23, XM69, XM70, XM71, XM72, XM84, XN52); Zamora (QF17, TL68). Portugal: Alto Alentejo (PD35); Beira Alta (NE69, PE48); Beira Baixa (PE33); Beira Litoral (NE45); Douro Litoral (NF47, NF85); Minho (NG72); Tras-os-Montes (PF59, PG83).

\section{Oenas Latreille, 1802}

El género Oenas se ha venido considerando representado en la Península Ibérica por tres especies: O. afer (Linnaeus, 1767), O. fusicornis Abeille de Perrin, 1880 y O. crassicornis (Illiger, 1800) (Górriz Muñoz, 1882; Rodríguez López-Neyra, 1914; Fuente, 1933; Pardo Alcaide, 1950). La presencia ibérica de la especie europea oriental $O$. crassicornis ha de considerarse errónea (Bologna, 1991). Por otra parte todos los individuos ibéricos examinados por nosotros corresponden morfológicamente a $O$. fusicornis, especie bien caracterizada, entre otros rasgos distintivos, por la forma y tamaño de los artejos antenales (Abeille de Perrin, 1880; Kaszab, 1951). Los ejemplares examinados de Pamplona ya portaban etiquetas de identificación manuscritas de A. Pardo Alcaide como O. fusicornis. A esta especie también corresponden los ejemplares detalladamente figurados por Valladares 


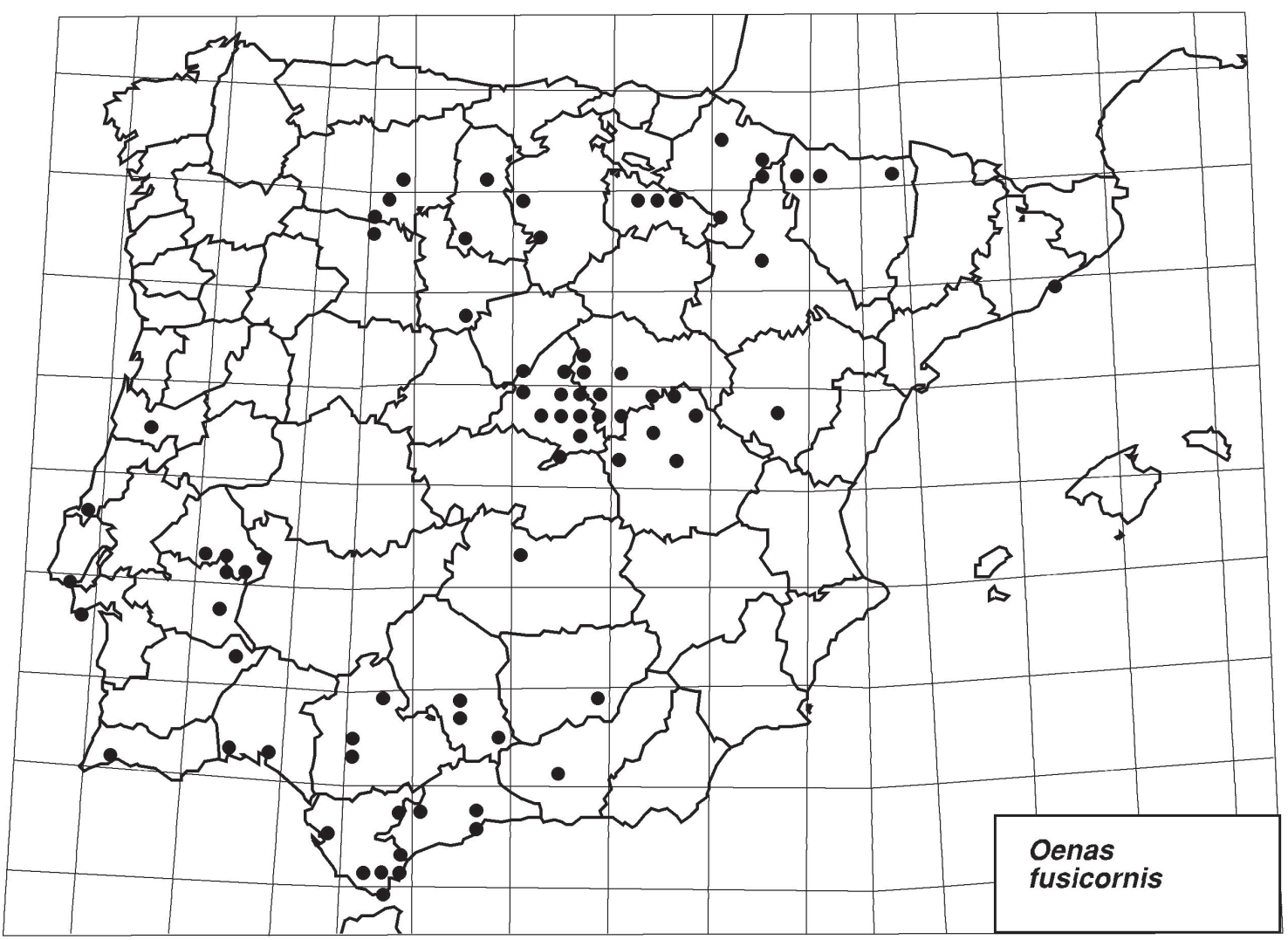

Fig. 5. - Mapa de la Península Ibérica con los puntos correspondientes a las localidades donde se ha localizado o citado en la bibliografía Oenas fusicornis Abeille de Perrin, 1880. Cada punto equivale a una cuadrícula UTM de 20 x $20 \mathrm{~km}$.

Fig. 5.- Map of the Iberian Peninsula with locality records for Oenas fusicornis Abeille de Perrin, 1880. Each dot represents a $20 \times 20 \mathrm{~km}$ UTM square.

Díez (1984) de León, aunque atribuidos por este autor a $O$. afer. Bologna (1991) señala que, aunque O. afer está citada de la mayor parte de la Península Ibérica, muchas de las localidades corresponden en realidad a $O$. fusicornis. Nosotros no sólo compartimos esta opinión, sino que, además, consideramos que la presencia de $O$. afer en la Península ha de considerarse dudosa y desde luego, requiere confirmación para poder ser incluida en el catálogo de especies ibero-baleares. Así, las citas ibéricas de $O$. afer (Linnaeus, 1758) probablemente se deban a confusiones con hembras de $O$. fusicornis, o bien al hecho de considerar a $O$. fusicornis como sinónimo de $O$. afer, siguiendo el criterio expresado por Borchmann (1917) y Mader (1927) en sus respectivos catálogos. Por último, aunque no se ha examinado el holotipo hembra de $O$. hispanus Abeille de Perrin, 1880, especie descrita de Andalucía ["Andalousie" (sic)] (Abeille de Perrin, 1880), los rasgos distintivos de esta supuesta especie parecen coincidir con los de la hembra de O. fusicornis por lo que provisionalmente, y siguiendo el criterio de otros autores (Rodríguez López-Neyra, 1914; Bologna, 1991) se considera un sinónimo de $O$. fusicornis.

\section{Oenas fusicornis Abeille de Perrin, 1880}

Especie descrita de Argelia ("Algérie", loc. typ.) y conocida de la Península Ibérica (Abeille de Perrin, 1880; Kaszab, 1951; Bologna, 1991). Aunque la descripción original de O. fusicornis es breve, el aspecto externo y la genitalia del macho de esta especie ya han sido presentados por Valladares Díez (1984), si bien con la denominación de $O$. afer. Especie de coloración poco variable, con los élitros y la cabeza de color negro y el 
protórax anaranjado-amarillento, a menudo con un reborde oscuro en la cara interna del borde posterior. La talla presenta una gran variabilidad intrapoblacional e interanual en una misma localidad.

En la Península Ibérica parece ocupar la mayor parte del territorio, desde el extremo sur hasta los Pirineos Occidentales. La fenología de los adultos se extiende desde finales de mayo hasta finales de julio, con el máximo de capturas en junio. En León los adultos todavía se encuentran activos en agosto (Valladares y Salgado, 1983).

Rosenhauer (1856) indica la presencia de $O$. afer en Algeciras y Granada. Salvañá Comas (1870) la cita bajo el nombre de $O$. afer de Mataró (Barcelona). Champion (1891) ya cita la presencia de $O$. fusicornis en Algeciras y Gibraltar. Martínez Sáez (1873) incluye la presencia de la especie como $O$. afer en Cuenca. Górriz Muñoz $(1881,1882$, 1902) recoge bajo la denominación de $O$. afer las citas previas de Amor en Córdoba, y añade Milagro (Navarra) y Lerma (Burgos). Oliveira (1894) cita la especie como O. afer de Coimbra y Marinha Grande. Medina (1895) cita $O$. afer de varios puntos de Sevilla. Rodríguez López-Neyra (1914) recoge las citas de Algeciras (Cádiz) y Gibraltar y bajo la denominación de $O$. afer de Andalucía, Navarra, Burgos, Mataró (Barcelona), Cuenca y Madrid. Fuente (1933) recoge registros previos y la cita como $O$. afer en Álava, Valladolid y La Rioja. Cobos (1949) cita $O$. afer en Málaga, pero el mismo autor (Cobos, 1958) rectifica la cita como $O$. fusicornis, basándose en determinaciones efectuadas por A. Pardo Alcaide. Serrano $(1981,1983)$ la señala como $O$. afer del P.N. da Arrábida y Santa Catarina (Estremadura) y recoge su presencia en Beira Litoral, Algarve, Alto Alentejo y Baixo Alentejo. Valladares \& Salgado (1983) y Valladares Díez (1984) citan la especie de varios puntos de León con la denominación de $O$. afer. Aguiar \& Serrano (1995) incluyen varias localidades del área de Cascais como $O$. afer. Pérez Moreno \& Cabrero Sañudo (1999) y Recalde et al. (2002) registran $O$. fusicornis en varias localidades de La Rioja y Aragón respectivamente.

MATERIAL EXAMINADO: Se han revisado 365 ejemplares de las siguientes localidades: ESPAÑA: BURGOS: Olmillos de Sasamón: 30-VI-1993, 4 ejemplares (P. Bahillo leg.) (MNCN). CADIZ: Algeciras: VI-1903, 2 ejemplares (Escalera leg.) (MNCN); 3-VI-1975, 5 ejemplares (J. de Ferrer leg.) (JF y JLR). Laguna de la Janda: 1 ejemplar (Cobos leg.) (EEZA). Laguna de Medina, Jerez: 3-VI-1982, 1 ejemplar (J. de Ferrer leg.) (JLR). San Roque: 5-VII-1971, 1 ejemplar (JF); 17-VII1971, 2 ejemplares (JF); 16-VI-1972, 2 ejemplares (JF); 8-VI1973, 3 ejemplares (J. de Ferrer leg.) (JF y JLR); VI-1979, 1 ejemplar (J. Ramírez leg.) (AZ). Tarifa: VI-1903, 1 ejemplar (Escalera leg.) (MNCN). Ubrique: 2-VI-1979, 2 ejemplares (J. de Ferrer leg.) (JLR). CIUDAD REAL: Las Casas: 13-VI1986, 4 ejemplares (JLZ). CORDOBA: Córdoba: 1 ejemplar (MNCN). El Canalizo, Luque: 28-VI-1998, 4 ejemplares (M. Baena leg.) (MB y JLR). Laguna del Salobral, Luque: 20-VII1984, 2 ejemplares (M. Baena leg.) (JLR). Santa Cruz: 11-V1997, 1 ejemplar (M. Baena leg.) (MB). CUENCA: Bólliga: 1VII-1982, 1 ejemplar (JLZ). Cañizares: 1 ejemplar (Selgas leg.) (MNCN). Cuenca: 1 ejemplar (MNCN). Rozalén del Monte: 4-VII-1995, 15 ejemplares (P. Barranco leg.) (JLR). Tragacete: 2 ejemplares (Selgas leg.) (MNCN); VII-1906, 2 ejemplares (Arias leg.) (MNCN). Tribaldos: 14-VI-2000, 1 ejemplar (M. García París leg.) (MNCN). Uclés: 1 ejemplar (MNCN). GRANADA: Carretera Granada-Sierra Nevada, 1300 m: 12-VI-1994, 5 ejemplares (J. L. Ruiz leg.) (JLR). Granada: VI-1909, 1 ejemplar (Escalera leg.) (MNCN). GUADALAJARA: Archilla: 8-VI-1984, 3 ejemplares (M. GarcíaParís leg.) (MNCN). Guadalajara: 1 ejemplar (MNCN). Pastrana: 1 ejemplar (MNCN). Pioz: 13-VI-1998, 5 ejemplares (M. García París leg.) (MNCN). Uceda: 1-VI-1985, 1 ejemplar (C. Rey leg.) (MNCN). Salmerón: 19-VII-1982, 1 ejemplar (JLZ). 2 km al E de Valdenuño: 30-VI-1998, 1 ejemplar (M. García París leg.) (MNCN). Zorita: 15-VI-1984, 3 ejemplares (C. Rey leg.) (MNCN). HUELVA: Ayamonte: V-1909, 1 ejemplar (Expedición del Museo) (MNCN). Huelva: 1 ejemplar (M. Vircanio leg.) (MNCN). La Ribera: 22-V-1985, 4 ejemplares (M. Huertas leg.) (SAE). HUESCA: Jaca: 2 ejemplares $(\mathrm{MNCN}) ; 2$ ejemplares (Arias leg.) (MNCN). Sierra de la Peña: 2 ejemplares (MNCN). JAEN: Cazorla: 23-VI-1916, 1 ejemplar (MNCN). Sierra de Cazorla: 8-VII-1973, 3 ejemplares (J. de Ferrer leg.) (JLR). LEÓN: La Bañeza: 17-VI-1991, Thapsia villosa, 1 ejemplar (P. Bahillo leg.) (MNCN). MADRID: Alto del Chaparral, Algete: 24-V-1999, 1 ejemplar (G. Astudillo leg.) (MNCN). Aranjuez: 1 ejemplar (MNCN); 1 ejemplar (G. Mercet leg.) (MNCN); VI-1906, 1 ejemplar (Arias leg.) (MNCN). Casa de la Aldehuela, $5 \mathrm{~km}$ al SO de Torrelaguna: 13-VI-1998, 5 ejemplares (M. García París leg.) (MNCN). 2 km al E de Carabaña: 13-VI-1998, 5 ejemplares (M. García París leg.) (MNCN). Cercedilla: Estación Alpina: III-1935, 1 ejemplar (J. Hernández leg.) (MNCN). El Escorial: 1 ejemplar (MNCN). Fresno de Torote: 3-VI-2000, 1 ejemplar (G. Astudillo leg.) (MNCN). Getafe: 1 ejemplar (MNCN). Loeches: 5 ejemplares (G. Carrasco leg.) (MNCN). Madrid: 1 ejemplar (MNCN); 1 ejemplar (G. Mercet) (MNCN). Montarco: 2 ejemplares (G. Mercet) (MNCN); 30-V-1897, 2 ejemplares (MNCN); VI-1908 (Arias leg.) (MNCN); 2 ejemplares (C. Bolívar leg.) (MNCN). Nuevo Baztán: 15-VI-1925, 5 ejemplares (MNCN); VI-1927, 3 ejemplares (MNCN). 3 km al NE de Olmeda de las Fuentes: 13-VI-1998, 5 ejemplares (M. García París leg.) (MNCN). Arroyo Vega, 1 km al O de Olmeda de las Fuentes: 8-VII-1998, 5 ejemplares (M. García París leg.) (MNCN). Perales de Tajuña: 13-VI-2000, 5 ejemplares (M. García París leg.) (MNCN). $3 \mathrm{~km}$ al O de Pezuela de las Torres: 29-VI-1998, 5 ejemplares (M. García París y C. Martín leg.) (MNCN). Ribatejada: 26-V-2000, 1 ejemplar (G. Astudillo leg.) (MNCN). Los Santos de la Humosa: 29-VI-1998, 5 ejemplares (M. García París y C. Martín leg.) (MNCN). 3 km al E de Los Santos de la Humosa: 29-VI-1998, 5 ejemplares (M. García París y C. Martín leg.) (MNCN). Tielmes: 14-VI-2000, 5 ejemplares (M. García París leg.) (MNCN). $2 \mathrm{~km}$ al S de Tielmes: 17-VI-2000, 1 ejemplar (M. García París leg.) (MNCN). $5 \mathrm{~km}$ al N de Tielmes: 15-VII-2000, 1 ejemplar (M. García París leg.) (MNCN). Torrelaguna - El Vellón: 5-VI- 
2000, 3 ejemplares (M. García París leg.) (MNCN). 3 km al SE de Torres de la Alameda: 30-VI-1998, 5 ejemplares (M. García París leg.) (MNCN). Valdelaguna: 10-VII-1998, 5 ejemplares (M. García París y C. Martín leg.) (MNCN). $3 \mathrm{~km}$ al O de Valdilecha: 10-VI-1998, 5 ejemplares (M. García París y C. Martín leg.) (MNCN). 5 km al O de Valdilecha: 10-VI-1998, 5 ejemplares (M. García París y C. Martín leg.) (MNCN). 3 km al SE de Villar del Olmo: 13-VI-1998, 5 ejemplares (M. García París leg.) (MNCN). Venturada: 15-VI-1985, 4 ejemplares (M. García París leg.) (MNCN). Villaviciosa [de Odón]: 10-VI1927, 1 ejemplar (MNCN). MALAGA: Málaga: 1 ejemplar (MNCN); 1 ejemplar (A. Sanz leg.) (MNCN); 2 ejemplares (Cobos leg.) (EEZA). Cazares [Casares]: 23-VI-1916, 1 ejemplar (MNCN). Refugio El Quejigar, Sierra de las Nieves, T.M. Parauta: 20-VI-1998, 35 ejemplares (J. L. Ruiz leg.) (JLR); 11VII-1998, 4 ejemplares (J. L. Ruiz leg. ) (JLR). Sierra de las Nieves, Ronda: 6-VII-1996, 1 ejemplar (J. de Ferrer leg.) (JLR). Pinsapar: 5-III-1984, 1 ejemplar (Barón leg.) (AZ). Torremolinos: 1 ejemplar (Cobos leg.) (EEZA). NAVARRA: Pamplona: 30-VII-1956, 8 ejemplares (S. V. Peris leg.) (MNCN). PALENCIA: Bárcena de Campos, 850 m: 11-VII1987, 1 ejemplar (P. Bahillo leg.) (MNCN). Castrillo de Villavega: 22-VI-1991, sobre Thapsia villosa, 2 ejemplares (P. Bahillo leg.) (MNCN). Villarramiel: 1 ejemplar (MZB) [en la etiqueta indica Orense]. SEVILLA: La Salud: VI-1941, 11 ejemplares (A. Benítez leg.) (MNCN). Sevilla: 1 ejemplar (MNCN); 3 ejemplares (Barras leg.) (MNCN). TERUEL: Teruel: 1 ejemplar (A. Sanz leg.) (MNCN). TOLEDO: Seseña: 1 ejemplar (MNCN). VALLADOLID: Olmedo: 1 ejemplar (MNCN). ZAMORA: Granucillo de Vidriales, Benavente, 850 m: 9-VII-1989, 3 ejemplares (P. Bahillo leg.) (MNCN). ZARAGOZA: Tiermas: 14 ejemplares (A. Sanz leg.) (MNCN). Sin datos: 23 ejemplares (MNCN). PORTUGAL: ALGARVE: Bensafrim: V-1909, 4 ejemplares (MNCN). Odiaxere: 15-V1982, 15 ejemplares (A. Zuzarte leg.) (AZ). Ría de Alvor: 14V-1982, 1 ejemplar (A. Zuzarte leg.) (AZ). ALTO ALENTEJO: Campomaior: 20-V-1986, 1 ejemplar (A. Zuzarte leg.) (AZ). Campomaior, Roças: 11-V-1990, 2 ejemplares, en umbelíferas (A. Zuzarte leg.) (AZ). Elvas, Correio-Mor: 28-V-1984, 8 ejemplares (A. Zuzarte leg.) (AZ). Elvas, Terrugem: 20-V1988, 1 ejemplar (A. Zuzarte leg.) (AZ). Fronteira: 30-V-1985, 6 ejemplares, en umbelíferas. (A. Zuzarte leg.) (AZ). Fronteira-Camparrão: 11-VI-1972, 1 ejemplar (A. Zuzarte leg.) (AZ). Monforte: Vaiamonte-Quinta: 24-V-1994, 3 ejemplares (A. Zuzarte leg.) (AZ). Redondo. Montoito. Mero. Casa Alta: 22-V-1990, 2 ejemplares (A. Zuzarte leg.) (AZ). Sto. Amaro-Sousel: 4-VI-1967, 1 ejemplar (A. Zuzarte leg.) (AZ); 5-VI-1968, 1 ejemplar (A. Zuzarte leg.) (AZ). BAIXO ALENTEJO: Moura-Safara. Merindade dos Lamiares: 2-V-1983, 1 ejemplar (A. Zuzarte leg.) (AZ). ESTREMADURA: Queluz: 7-V-1984, 1 ejemplar (A. Zuzarte leg.) (AZ); 8-V-1985, 1 ejemplar (A. Zuzarte leg.) (AZ). Río-de-Mouro: 10-V-1962, 1 ejemplar (L. Ambar leg.) (AZ).

Los ejemplares examinados y citas bibliográficas corresponden a las siguientes provincias y cuadrículas UTM de 10x10 km: España: Barcelona (DF59); Burgos (VM19, VM35); Cádiz (QA65, TE68, TF41, TF70, TF80, TF81, TF86); Ciudad Real (VJ12), Córdoba (UG49, UG57, UG85, UG95); Cuenca (WK02, WK12, WK55, WK68, WK73, WK96); Granada (VG41, VG51); Guadalajara (VK87, VK89, VL60, VL62, WK06, WK07, WK48, WL00); Huelva (PB42, PB82); Huesca (BH90, XN90, YN01); Jaén (VG99); La Rioja
(WM68, WM39, WM49); León (TM57, TM68, TN80, TN90); Madrid (VK09, VK26, VK36, VK43, VK47, VK56, VK59, VK64, VK67, VK69, VK75, VK76, VK77, VK78, VK86, VK87, VK88, VL11, VL41, VL51, VL60); Málaga (TF82, UF16, UF65, UF76); Navarra (XM07, XN14); Palencia (UM45, UN70) Sevilla (TG33, TG34, TG69); Teruel (XK66); Toledo (VK43); Valladolid (UL57); Zamora (TM55); Zaragoza (XM52, XN40, XN52). Gibraltar (TF80). Portugal: Algarve (NB21, NB30); Alto Alentejo (PC27, PD12, PD21, PD22, PD50, PD62); Baixo Alentejo (PC42); Beira Litoral (NE45); Estremadura (MC68, MC78, MC79, MC95, MD96, NE00).

\section{Physomeloe Reitter, 1911}

El género Physomeloe transferido recientemente a la tribu Lyttini debido a sus caracteres larvarios (Bologna y Aloisi, 1994) incluye una única especie, endémica de la Península Ibérica.

\section{Physomeloe corallifer (Germar, 1818)}

Especie de coloración homogénea, excepto ligeras variaciones en la coloración de los tubérculos protorácicos (Flach, 1907), y talla muy variable, bien distribuida en el centro de Portugal y la meseta española (Extremadura, Castilla-León, CastillaLa Mancha, Madrid) y sólo representada por escasas citas en Andalucía (Bologna y Aloisi, 1994).

La mayoría de los ejemplares examinados fueron colectados de marzo a mayo, con una única cita registrada en julio en el Sistema Central. Exceptuando la captura de julio todos los demás ejemplares entran dentro de la fenología indicada por autores anteriores (Górriz Muñoz, 1882; Valladares \& Salgado, 1983; Bologna \& Aloisi, 1994). La especie alcanza al menos $1600 \mathrm{~m}$ de altitud en el Sistema Central (Puerto de la Morcuera y Puerto de Guadarrama).

Górriz Muñoz (1882) recoge las citas previas de Amor en Córdoba, y las de Graells de la provincia de Madrid, y añade Aranda de Duero, Lerma y Tardajos (Burgos) y Madrid (Madrid). Oliveira (1894) cita la especie de la Serra de Montesinho, Oliveira do Hospital, Valle d'Azares, Guarda, Freineda y Serra da Estrella. Reitter (1895) cita la especie de Portugal sin precisar. Traizet (1896) la cita en Rivas (Madrid). Flach (1907) en Madrid. Rodríguez López-Neyra (1914) presenta citas generales en Andalucía, Castilla y Extremadura. Redondo (1915) en los alrededores de Salamanca. Iglesias (1928) cita la especie en Vigo (Pontevedra). Sumacov (1934) recoge la presencia de la especie en Vicálvaro (Madrid). Torres-Sala (1962) vuelve a 


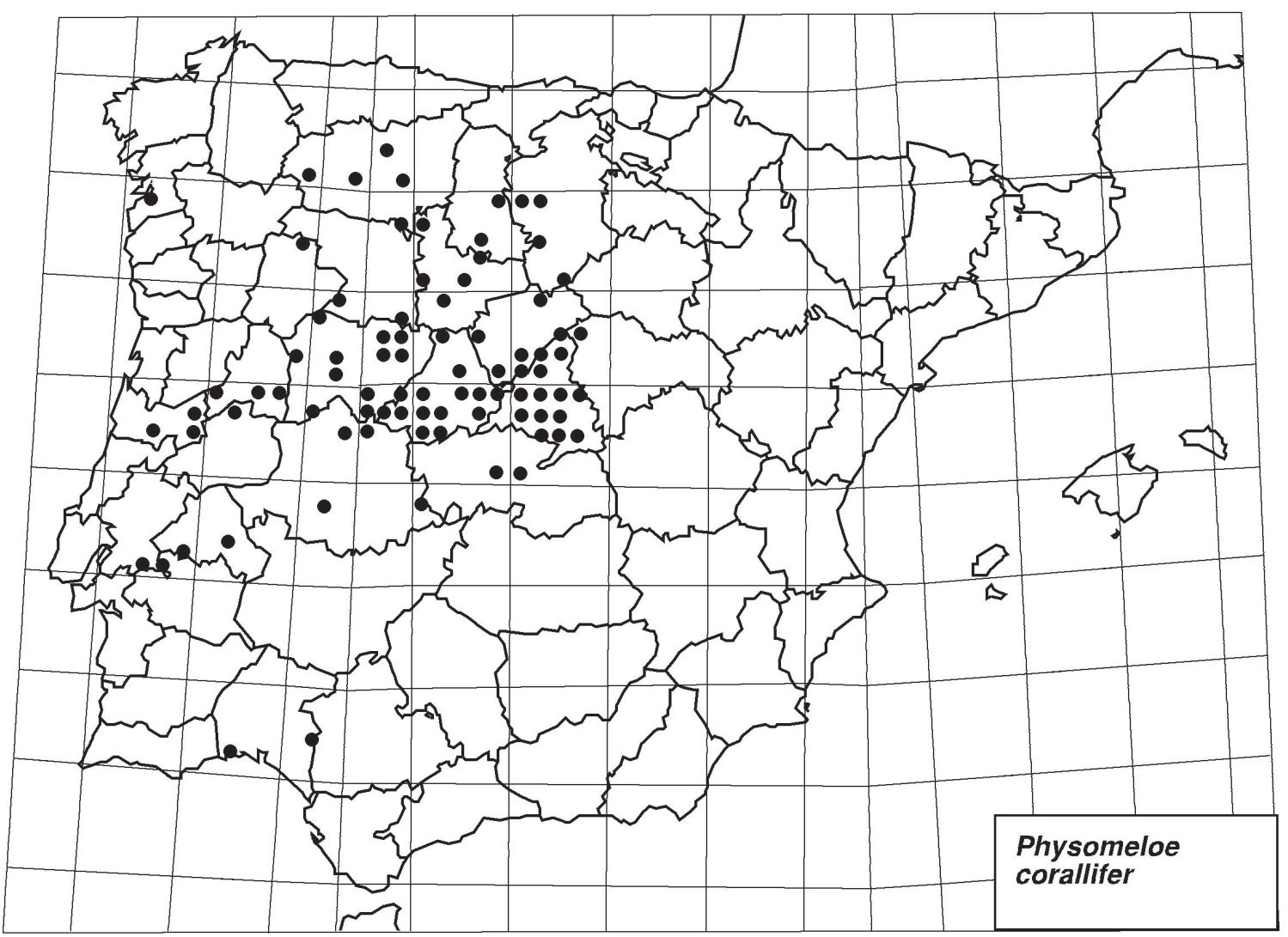

Fig. 6.- Mapa de la Península Ibérica con los puntos correspondientes a las localidades donde se ha localizado o citado en la bibliografía Physomeloe corallifer (Germar, 1818). Cada punto equivale a una cuadrícula UTM de 20 x $20 \mathrm{~km}$.

Fig. 6.- Map of the Iberian Peninsula with locality records for Physomeloe corallifer (Germar, 1818). Each dot represents a 20 x $20 \mathrm{~km}$ UTM square.

citarla de Madrid. Valladares y Salgado (1983) y Valladares Díez (1984) citan la especie de varios puntos de León. Bologna \& Aloisi (1994) relacionan las siguientes localidades: Avila, El Tiemblo, Gredos, 15 km SW Navacepeda de Tormes, Madrigal de las Altas Torres, Poyales del Hoyo, Sotillo de la Adrada y $5 \mathrm{~km} \mathrm{~N}$ Venta del Obispo (Avila), Torrequemada (Cáceres), Motril (Granada), Astorga, Irede, Las Médulas, León, Mora de Luna y Valderas (León), Cercedilla, Cienvallejos, El Escorial, Guadarrama, Lozoya, Madrid, Collado Mediano, Miraflores de la Sierra, Navacerrada y Puerto de la Morcuera (Madrid), Palencia (Palencia), Candelario, Negrilla y $10 \mathrm{~km}$ N Salamanca (Salamanca), San Rafael y San Udefo [San Ildefonso] (Segovia), Montalbán (Teruel), Toledo e Illescas (Toledo), Granja Muedra cerca de Valoria la Buena, Tordesillas y La Unión (Valladolid) y las localidades portuguesas previa- mente recogidas por Oliveira (1894). Ortuño \& Hernández (1993) la citan de Patones (Madrid) a propósito de una malformación.

Consideramos que la cita de "Montalbán" (Bologna y Aloisi, 1994; Recalde et al., 2002) es mucho más probable que corresponda al área de $\mathrm{La}$ Puebla de Montalbán en la provicia de Toledo, donde la especie es frecuente, que a una localidad de la provincia de Teruel, donde ecológicamente la presencia de la especie es poco probable. Existe además una cita de la especie en Motril (Granada) (Bologna y Aloisi, 1994) y dos ejemplares, uno en la Colección del MNCN con etiqueta de "Almería", y otro en la colección de la Universidad de Alicante de "Torremanzanas" (Alicante) IV-1996 (J. V. Toledo leg.), que se encuentran muy alejados geográficamente del resto de los ejemplares citados o examinados. Por lo tanto considermos que la presencia de $P$. corallifer en Aragón, Valencia y 
Andalucía Oriental, aunque no descartable, necesita nueva documentación y de momento, estos puntos no se incluyen en los mapas de distribución.

MATERIAL EXAMINADO: Se han revisado 342 ejemplares recogidos en: ESPAÑA: AVILA: Arenas de San Pedro: 1 ejemplar (J. Ardois leg.) (MNCN). Arenas de San Pedro, Río Pelayo: 27-II-2000, 1 ejemplar (M. García París leg.) (MNCN). El Barco de Ávila: 5-IV-1999, 1 ejemplar (M. García París y G. Parra leg.) (MNCN). Candeleda: 17-VII-1984, 1 ejemplar (M. J. García leg.) (USal). Gredos: 2 ejemplares (J. Ardois leg.) (MNCN). La Plataforma, Hoyos del Espino: 5-IV-1999, 1 ejemplar (M. García París y G. Parra leg.) (MNCN). La Adrada: 10-IV-1985, 1 ejemplar (E. Rodríguez leg.) (USal). Madrigal [de las Altas Torres]: 2 ejemplares (J. Ardois leg.) (MNCN). Mombeltrán: 2 ejemplares (J. Ardois leg.) (MNCN). Navalmoral: 12-IV-1985, 1 ejemplar (M. L. Martín leg.) (USal). $1 \mathrm{~km}$ al S de Navalperal de Tormes: 5-IV-1999: 1 ejemplar (M. García París y G. Parra leg.) (MNCN). Pedro Bernardo, Gredos: VI-1966, 1 ejemplar (Esp. Nèg. leg.) (MZB). Cueva Valiente, Peguerinos: 4-IV-1999, 1 ejemplar (M. García París y G. Parra leg.) (MNCN). Poyales: 2 ejemplares (J. Ardois leg.) (MNCN). Poyales del Hoyo: 19-IV1985, 2 ejemplares (M. de los Mozos leg.) (JLR). Vertiente N del Puerto Serranillos: 27-II-2000, 1 ejemplar (M. García París leg.) (MNCN). Puerto de Villatoro, 1356 m: 26-V-1989, 1 ejemplar (A. Zuzarte leg.) (AZ). San Martín de la Vega del Alberche: 1-III-1997, 1 ejemplar (J. L. Zapata leg.) (JLZ). Sotillo de la Adrada: 2 ejemplares (L. Velasco leg.) (MNCN). El Tiemblo: 5-IV-1999, 2 ejemplares (J. R. Verdú y E. Micó leg.) (MNCN). Villarejo [del Valle]: 1 ejemplar (MNCN). BURGOS: Moradillo de Roa: 17-V-2000, 1 ejemplar (M. García París leg.) (MNCN). Olmillos de Sasamon: 8-IV-1996, 2 ejemplares (P. Bahillo leg.) (MNCN). CACERES: Hervás: 16-IV-1983, 1 ejemplar (J. Serra leg.) (USal); 24-III-1987, 1 ejemplar (M. A. García leg.) (USal); 24-IV-1987, 1 ejemplar (L. C. Rojo leg.) (USal); 24-IV-1987, 1 ejemplar (P. O'Mullony leg.) (USal); 25-IV-1987, 1 ejemplar (C. Andreu leg.) (USal); 29-IV-1987, 1 ejemplar (B. Santos leg.) (USal); 29-IV-1987, 1 ejemplar (M. J. Carpio leg.) (USal); 29-V-1987, 1 ejemplar (J. Montero leg.) (USal); 30-IV-1987, 1 ejemplar (C. Vaquero leg.) (USal); 4-V-1987, 1 ejemplar (G. Moreno leg.) (USal); IV-1987, 1 ejemplar (C. García leg.) (USal). Jaraíz: 15-IV-1987, 1 ejemplar (F. Hannemann leg.) (USal). Jerte: 15-IV-1985, 1 ejemplar (E. Pérez leg.) (USal). Puerto de Honduras, SE de Hervás: 14-V-2000, 1 ejemplar (M. García París leg.) (MNCN). Pantano Gabriel y Galán: 29-IV1987, 1 ejemplar (A. A. García leg.) (USal). Pto. S. Vicente: 2-VI-1966, 1 ejemplar (Esp. Nèg. leg.) (MZB). GUADALAJARA: Puente de El Cardoso de la Sierra: 1-V-1999, 2 ejemplares (S. Martín leg.) (MNCN). HUELVA: Ayamonte: V1909, 1 ejemplar (Expedición del Museo) (MNCN). Santana [Escacena del Campo]: 23,31-III-1985, 1 ejemplar (I. Izquierdo leg.) (MNCN). MADRID: Alcalá de Henares: 20III-1999, 1 ejemplar (G. Astudillo leg.) (MNCN). Alto del Chaparral, Algete: 24-V-1999, 1 ejemplar (G. Astudillo leg.) (MNCN). Becerril de la Sierra: 5-III-2000, 28 ejemplares (F. A. Montes leg.) (MNCN). Brunete: Cienvallejos: 1 ejemplar (MNCN). Cercedilla: 1 ejemplar (J. Ardois leg.) (MNCN); 1 ejemplar (S. V. Peris leg.) (MNCN); 1 ejemplar (G. Mercet leg.) (MNCN); 1 ejemplar (Bolívar leg.) (MNCN); 2 ejemplares (Expedición del Museo) (MNCN); 1 ejemplar (Moroder leg.) (MZB); 1 ejemplar (Exp. del Museo) (MZB); 2 ejemplares (M. Hernández leg.) (MNCN); 4 ejemplares (C. Bolívar leg.) (MNCN); 11 ejemplares (G. Schramm leg.) (MNCN). Cercedilla: La Peñota: 19-IV-1945, 1 ejemplar (S. V. Peris leg.) (MNCN). Pradera de Navalurraque, Cercedilla: 18-IV1999, 1 ejemplar (F. A. Montes leg.) (MNCN). Puerto de la Fuenfría, Cercedilla: 10-IV-1999, 4 ejemplares (F. A. Montes leg.) (MNCN). El Ventorrillo, Cercedilla: 22-III-1998, 1 ejemplar (F. A. Montes leg.) (MNCN); 28-II-1999, 7 ejemplares (F. A. Montes leg.) (MNCN). Ventorrillo: VI-1946, 4 ejemplares (Mateu leg.) (MZB). Canto Blanco: 17-III-1976, 1 ejemplar (C. Rey leg.) (MNCN); 21-IV-1976, 1 ejemplar (C. Rey leg.) (MNCN); 8-III-1977, 1 ejemplar (C. Rey leg.) (MNCN). Cerro San Pedro: 21-II-1993, 1 ejemplar (JLZ). Chinchón: 10-V-1979, 1 ejemplar (M. A. Sobrino leg.) (MAS). Collado Mediano: 2 ejemplares (Moróder leg.) (MNCN). Ermita del Espino, Daganzo: 8-V-1999, 1 ejemplar (G. Astudillo leg.) (MNCN). El Escorial: 1 ejemplar (Bolívar leg.) (MNCN); 1 ejemplar (Arias leg.) (MNCN); 2 ejemplares (Bolívar leg.) (MNCN); 2 ejemplares (M. Escalera leg.) (MNCN); 4 ejemplares (C. Bolívar leg.) (MNCN); 1040 m, 1 ejemplar (J. Lauffer leg.) (MNCN). Fuente la Teja, El Escorial: 4-IV-1999, 2 ejemplares (M. García París y G. Parra leg.) (MNCN). Gózquez de Abajo: 16-III-2000, 1 ejemplar (M. García París leg.) (MNCN). Guadarrama: Alto de los Leones: V-1965, 2 ejemplares (MNCN). Puerto de los Leones, Guadarrama: 8-III-1998, 1 ejemplar (F. A. Montes leg.) (MNCN). $2 \mathrm{~km}$ al O de La Cabrera: 20-II-2000, 1 ejemplar (M. García París leg.) (MNCN). Las Rozas: 11-IV-1959, 2 ejemplares (Gras leg.) (MZB). La Pedriza de Manzanares: 13III-1982, 1 ejemplar (M. Ortego leg.) (AZ). Los Molinos: 5V-1953, 1 ejemplar (S. V. Peris leg.) (MNCN); 5-V-1953, 1 ejemplar (W. Steiner leg.) (MNCN). Los Rancajales: 16-II1996, 1 ejemplar (J. L. Zapata leg.) (JLZ); 25-II-1996, 1 ejemplar (J. L. Zapata leg.) (JLZ). Lozoya: 1 ejemplar (Bolívar leg.) (MNCN). Madrid: 6 ejemplares $(\mathrm{MNCN}) ; 1$ ejemplar (Dusmet leg.) (MNCN); 1 ejemplar (E. Pons leg.) (MNCN); 1 ejemplar (S. V. Peris leg.) (MNCN); 3 ejemplares (Bolívar leg.) (MNCN); 1 ejemplar (G. Schramm leg.) (MNCN); 2 ejemplares (Arias leg.) (MNCN); 3 ejemplares (A. Sanz leg.) (MNCN); 2 ejemplares (A. Sanz leg.) (MZB). 14 ejemplares (J. Ardois leg.) (MNCN); V-1908, 1 ejemplar (L. Hernández leg.) (MNCN); 16-V-1925, 1 ejemplar (Col. Español) (MZB). Madrid: Canal: 14-III-1906, 1 ejemplar (Arias leg.) (MNCN). Madrid: Cerro Almodóvar: 7-III-1987, 2 ejemplares (JLZ). Madrid: Chamartín: 3 ejemplares (S. V. Peris leg.) (MNCN). Madrid: Ciudad Universitaria: 21-III-1943, 2 ejemplares (MNCN); 29-III-1954, 2 ejemplares (A. García Velázquez leg.) (MNCN). Madrid: Dehesa de la Villa: 1 ejemplar (S. V. Peris leg.) (MNCN); La Dehesa [de la Villa]: 27-III-1988, 1 ejemplar (JLZ); 15-V-1988, 1 ejemplar (JLZ); D. [Dehesa de la] Villa: 16-V-1980, 1 ejemplar (M. A. Sobrino leg.) (MAS). Madrid: El Pardo: 21-III-1957, 1 ejemplar (S. V. Peris leg.) (MNCN). Madrid: Vallecas: 4-IV-1954, 1 ejemplar (W. Steiner leg.) (MNCN). Madrid: Valdebernardo, Vicálvaro: 10IV-1998, 1 ejemplar (C. Martín leg.) (MNCN). Madrid: Virgen del Cortijo: 9-III-1977, 1 ejemplar (C. Rey leg.) (MNCN); 27-III-1977, 1 ejemplar (C. Rey leg.) (MNCN). Miraflores de la Sierra: 4 ejemplares (Expedición del Museo) (MNCN). Puerto de la Morcuera: 1600 m, 21-IV-1984, 1 ejemplar (C. Rey leg.) (MNCN). Navacerrada: 1 ejemplar (Arias leg.) (MNCN). Peña Pintada, Navacerrada: 11-IV- 
1999, 1 ejemplar (F. A. Montes leg.) (MNCN). Puerto de la Morcuera: 8-III-1998, 1 ejemplar (F. A. Montes leg.) (MNCN). Rascafría: 10-III-2000, 5 ejemplares (M. García París leg.) (MNCN). Puerto de Malagón, Santa María de la Alameda: 3-V-1998, 1 ejemplar (F. A. Montes leg.) (MNCN). Valdemanco [de la] Sierra: 1 ejemplar (MNCN). $2 \mathrm{~km}$ al S de Valdemanco: 20-II-2000, 1 ejemplar (M. García París leg.) (MNCN). PALENCIA: Osorno: 21-VI-1984, 1 ejemplar (L. A. Fuente leg.) (USal). SALAMANCA: Amatos de Alba: 3 IV-1999, 1 ejemplar (M. García París y C. Martín leg.) (MNCN). Béjar: 24-IV-1985, 1 ejemplar (M. C. Alvarez leg.) (USal); 18-IV-1987, 1 ejemplar (A. Redondo leg.) (USal); 24 III-1995, 1 ejemplar (B. Velasco leg.) (USal). Boadilla: $1 \mathrm{~km}$ al E: 3-IV-1999, 1 ejemplar (M. García París y C. Martín leg.) (MNCN); 3 km al E: 3-IV-1999, 1 ejemplar (M. García París y C. Martín leg.) (MNCN); 4 km al E: 3-IV-1999, 1 ejemplar (M. García París y C. Martín leg.) (MNCN). Carretera de Boadilla - San Muñoz: 3-IV-1999, 1 ejemplar (M. García París y C. Martín leg.) (MNCN). Cabrerizos: 24-IV-1983, 1 ejemplar (H. López leg.) (USal). Candelario: 24-III-1985, 1 ejemplar (M. L. Caballero leg.) (USal). Castillo del Buen Amor: 18-IV-1985, 1 ejemplar (A. Martín Pérez leg.) (USal). Florida de Liébana: 10-V-1987, 1 ejemplar (M. J. Gómez leg.) (USal). La Flecha: 18-VII-1984, 1 ejemplar (M. Alberto leg.) (USal); 12-IV-1985, 1 ejemplar (M. Hernández leg.) (USal); 18-X-1985, 1 ejemplar (Valle Benito leg.) (USal). Linares de Riofrío: 14-IV-1985, 1 ejemplar (J. M. Fdz. Prieto leg.) (USal). Linares R. [Riofrío]: 8-IV-1985, 1 ejemplar (C. Vicente leg.) (USal). Los Santos: 3-IV-1985, 1 ejemplar (P. Hernández leg.) (USal). Lumbrales: 25-VII-1984, 1 ejemplar (M. A. Chico leg.) (USal). Negrilla: 9 ejemplares (Escribano leg.) (MNCN). Pereña: 1-V-1986, 1 ejemplar (P. Bahillo leg.) (MNCN). Pte. de la Salud: 1 ejemplar (I. Rucino leg.) (USal); 29-III-1985, 2 ejemplares (Ga. López-Dóriga leg.) (USal) Retortillo: 10-IV-1985, 1 ejemplar (O. J. S. leg.) (USal); 20IV-1985, 1 ejemplar (P. Domingo leg.) (USal). Salamanca, cerca de la Fuente del Cántaro: 15-IV-1985, 1 ejemplar (R Villamor leg.) (USal). Salamanca: 13-IV-1985, 1 ejemplar (E. Lage leg.) (USal); 16-V-1986, 1 ejemplar (Grupo G leg.) (USal); 17-IV-1995, 1 ejemplar (F. J. San Millán leg.) (USal); 19-IV-1985, 1 ejemplar (J. L. Martín Gómez leg.) (USal); $19-$ IV-1985, 1 ejemplar (J. L. Santiago leg.) (USal); 20-III-1985, 1 ejemplar (C. Fresno leg.) (USal); 22-IV-1985, 1 ejemplar (F. J. Esteban leg.) (USal); 23-IV-1985, 1 ejemplar (J. J. García leg.) (USal); 27-IV-1985, 1 ejemplar (A. Romano leg.) (USal); 28-III-1985, 1 ejemplar (A. Marco leg.) (USal); 29IV-1986, 1 ejemplar (M. R. Melerdo leg.) (USal); 30-III-1985, 1 ejemplar (A. Alonso leg.) (USal); 3-IV-1985, 1 ejemplar (A. Muñoz leg.) (USal); 4-III-1982, 1 ejemplar (M. Milicuo leg.) (USal); IV-1985, 1 ejemplar (E. Torres leg.) (USal); V-1987, 1 ejemplar (J. R. García leg.) (USal). San Miguel [de Valero]: 13-IV-1985, 1 ejemplar (C. Vigarte leg.) (USal). San Miguel de Valero: 27-IV-1985, 1 ejemplar (A. I. Prado leg.) (USal). Sierra de Béjar: 24-III-1983, 1 ejemplar (P. Bragado leg.) (USal). Tejares [Salamanca]: 17-III-1985, 1 ejemplar (M. A Hernández leg.) (USal). SEGOVIA: El Espinar: 1 ejemplar (J. Ardois leg.) (MNCN); 1-V-2000, 1 ejemplar (F.A. Montes leg.) (MNCN). Sabinar de Prádena: 23-V-1998, 1 ejemplar (J. París leg.) (MNCN). La Granja: V-1906, 2 ejemplares (MZB). San Ildefonso: VII-1902, 1 ejemplar (MNCN). San Rafael: 2 ejemplares (C. Bolívar leg.) (MNCN). Santiuste [de San Juan Bautista]: 29-III-1985, 1 ejemplar (J. T. de Nimlán leg.)
(USal). Segovia: IV-1957, 2 ejemplares (Español leg.) (MZB). TOLEDO: Toledo: 7-IV-1989, 1 ejemplar (S. Sobrino leg.) (MAS). VALLADOLID: Tiedra: 22-V-1984, 1 ejemplar (A. Jiménez leg.) (USal). Valladolid: 1 ejemplar (MZB); Parquesol: 26-III-1997, 1 ejemplar (P. Bahillo leg.) (MNCN). ZAMORA: $3 \mathrm{~km}$ al NO de Fuentesaúco: 9-V-1998, 1 ejemplar (M. García París y C. Martín leg.) (MNCN). Zamora: 9IV-1982, 1 ejemplar (T. Esteban leg.) (USal). PORTUGAL: ALTO ALENTEJO: Barroca-Mora: 13-III-1968, 1 ejemplar (A. Zuzarte leg.) (AZ). Mora: 12-III-1968, 1 ejemplar (A. Zuzarte leg.) (AZ). Serra São Mamede, Portalegre: 8-III1992, 1 ejemplar (A. Zuzarte leg.) (AZ). BEIRA ALTA: Colmeal da Serra: 16-IV-1964, 1 ejemplar (AZ). Gouveia: 1VI-1983, 1 ejemplar (T. Branco leg.) (AZ). Serra da Estrela: 1 ejemplar (MNCN). RIBATEJO: Coruche: 5-III-1977, 1 ejemplar (A. Zuzarte leg.) (AZ); 11-IV-1982, 1 ejemplar (A. Zuzarte leg.) (AZ). Coruche-Bairro da Serraçás: 26-III-1977, 1 ejemplar (A. Zuzarte leg.) (AZ). Coruche-Boichimos: 9-IV1967, 1 ejemplar (A. Zuzarte leg.) (AZ); 30-V-1967, 1 ejemplar (A. Zuzarte leg.) (AZ). Coruche-Fajarda: 23-II-1975. 1 ejemplar (F. Ferreira leg.) (AZ); 31-III-1979, 1 ejemplar (A. Zuzarte leg.) (AZ). Coruche-Monte da Barca: 20-III-1983, 4 ejemplares (A. Zuzarte leg.) (AZ); 7-IV-1981, 5 ejemplares (C. Zuzarte leg.) (AZ). Galveias: 6-IV-1977, 1 ejemplar (T. Branco leg.) (AZ).

Se han observado ejemplares, no capturados, en: MADRID: Manjirón, VL5136: 30-III-2002, 1 ejemplar (I. Martínez-Solano). SALAMANCA: "El Baldío" de Martiago, 29T QE1573, 940 m: 31-III-02, 3 ejemplares (J. Serradilla).

Los ejemplares examinados y citas bibliográficas corresponden a las siguientes provincias y cuadrículas UTM de 10x10 km: España: Avila (TK87, UK04, UK05, UK06, UK14, UK17, UK18, UK25, UK26, UK27, UK35, UK36, UK58, UK66, UK77, UK79, UK99, UL35, UL50); Burgos (VL39, VM19, VM35, VM38, VM41); Cáceres (QD36, QE45, TK36, TK44, TK56, UJ17); Guadalajara (VL64); Huelva (PB42, QB35); León (PH80, QH40, TM96, TN64, TN81); Madrid (UK89, VK09, VK17, VK28, VK36, VK37, VK38, VK46, VK47, VK48, VK55, VK57, VK59, VK64, VK68, VL00, VL01, VL10, VL11, VL20, VL21, VL22, VL30, VL31, VL32, VL33, VL42, VL52, VL53); Palencia (UM75, UM88); Pontevedra (NG27); Salamanca (PF93, QE17, QF06, QF22, QF31, TK57, TK59, TK67, TK89, TL64, TL73, TL74, TL75, TL83, TL85, TL92); Segovia (UL65, UL90, UL91, VL03, VL12, VL45); Toledo (UK81, VK11, VK24); Valladolid (UL39, UM00, UM06, UM40, UM51, UM62); Zamora (TL68, TL86). Portugal: Alto Alentejo (ND70, ND71, PD35); Beira Alta (NE84, NE96, PE18, PE27, PE48, PE79, PK59); Beira Litoral (NE45); Ribatejo (ND41, ND83); Tras-osMontes (PG84).

\section{Conclusiones}

Tras el examen de 1955 ejemplares ibéricos de Lyttini consideramos que la fauna de esta tribu en la Península Ibérica queda reducida a seis especies, dos del género Berberomeloe y una en cada uno de los géneros Lagorina, Lytta, Oenas y Physomeloe. 
El género Physomeloe con un único representante, $P$. corallifer, y de relaciones filogenéticas inciertas (Bologna y Pinto, 2002), es endémico de la Península Ibérica. Physomeloe corallifer se distribuye por casi toda la mitad occidental peninsular, con penetraciones a lo largo del Sistema Central, aunque faltan citas en A Coruña, Lugo, Ourense, Asturias, Badajoz, Ciudad Real, Guadalajara, Córdoba, Sevilla y algunas regiones de Portugal. Su límite de distribucción oriental parece marcado por la presencia dominante de sustratos calcáreos o de yesos en zonas relativamente secas, si bien existen algunas localidades en las que se ha encontrado sobre este tipo de suelos (Vicálvaro, Rivas, Gózquez). Las 4 citas de Teruel, Almería, Granada y Alicante, aunque no descartables, requieren confirmación, ya que son los únicos datos de la presencia de esta llamativa y conspicua especie en el este peninsular.

Oenas fusicornis es la única especie del género que hemos podido localizar en España y Portugal. Sus citas se encuentran bien distribuidas por casi todo el territorio peninsular, aunque faltan en: Galicia, Asturias, Cantabria, la mayor parte de Cataluña y País Vasco, Extremadura, Comunidad Valenciana, Zamora y algunas regiones portuguesas. Es posible que la ausencia de la especie en gran parte de la Cornisa Cantábrica y Cataluña sea real, pero probablemente se encuentre en todo el resto peninsular.

Otra especie endémica de la península es Berberomeloe insignis, un endemismo del sureste español, únicamente registrada en 24 cuadrículas UTM de 10 x $10 \mathrm{~km}$. Se trata de la especie ibérica de la tribu con distribución más restringida, presente en zonas de Almería, Granada y Murcia. Parece muy probable que esta especie se encuentre amenazada por la proliferación de explotaciones agrícolas intensivas, campos de golf y por el uso de fertilizantes y pesticidas en su reducida área de distribución.

Berberomeloe majalis tiene una amplia distribución ibérica, y penetra en Francia y en varios puntos del norte de Africa. Faltan citas de la especie en Asturias, Cantabria y la mayor parte de Galicia y País Vasco, donde la presencia de la especie es posible, sobre todo en valles interiores secos.

Lagorina sericea, una especie que también se encuentra en el noroeste de África, se encuentra bien distribuida por la provincia de Cádiz, con penetraciones adicionales en Córdoba, Málaga, Sevilla y Huelva. Lytta vesicatoria es una especie con amplia distribución eurosiberiana. En Iberia su distribución se extiende por toda la mitad septen- trional, con la Serra de São Mamede (Portugal) como límite meridional. Faltan citas en algunas regiones de Portugal, en el País Vasco, en Cantabria, Lugo, Valladolid y Soria.

\section{AGRADECIMENTOS}

Agradecemos a A. J. Zuzarte, J. de Ferrer, P. Bahillo, J. L. Zapata, M. Baena, M. A. Sobrino y a la Sociedad Entomológica Andaluza el poner a nuestra disposición sus respectivas colecciones de meloidos. A G. Astudillo, J. Kohler, I. Martínez-Solano y J. Serradilla por comunicarnos amablemente sus observaciones de $B$. insignis y $P$. corallifer. A L. Capote y E. Recuero su ayuda en la localización de cuadrículas UTM. A M. A. Alonso-Zarazaga la revisión crítica del manuscrito y la localización geográfica de algunas localidades difíciles. A E. Galante, E. Micó y J. R. Verdú el facilitarnos la revisión de la colección de la Universidad de Alicante; a S. Férnandez Gayubo y M. Lizana por las facilidades prestadas para la revisión de la colección de la Universidad de Salamanca; a G. Masó su amabilidad durante la revisión de la colección del Museu de Zoología de Barcelona; a C. Martín su ayuda en la revisión del material de la colección del Museo Nacional de Ciencias Naturales (CSIC). Agradecemos a las Consejerías de Medio Ambiente de Andalucía, Castilla-La Mancha, Castilla-León y Madrid la concesión de autorizaciones de captura científica cuando ha sido necesario. Este trabajo se ha beneficiado de los recursos proporcionados por el proyecto D.G.I.: REN20001541/GLO.

\section{Referencias}

Abeille de Perrin, E., 1880. Contribution à la Faune Coléoptérologique de la Méditerrannée. Société d'Historie Naturelle de Tolouse, 14: 233-262.

Aguiar, C. A. S. \& Serrano, A. R. M., 1995. Estudo faunístico e ecológico dos Coléopteros (Insecta, Coleoptera) do Concelho de Cascais (Portugal). Boletim da Sociedade Portuguesa de Entomologia, 6(5): 41-66.

Asso, I. J. DE, 1784. Introductio in Oryctographiam et Zoologiam Aragoniae. Accedit Enumeratio stirpium in eadem regione noviter detectarum. Classis V. Insecta. Ordo I. Coleoptera. pp. 96-109. En Catalogus de la Entomofauna Aragonesa, 7: 13-16. (Obra original no consultada).

BolognA, M. A., 1989. Berberomeloe, a new west Mediterranean genus of Lyttini for Meloe majalis Linné (Coleoptera, Meloidae). Systematics and bionomics. Bollettino di Zoologia, [1988], 55: 359-366.

Bologna, M. A., 1991. Fauna de Italia. Coleoptera Meloidae. Calderini. Bologna. 541 pp.

Bologna, M. A. \& Aloisi, G., 1994. Systematics and bionomics of Physomeloe Reitter, 1911, with description of the first instar larva (Coleoptera, Meloidae). Eos, 69: 45-56. 
Bologna, M. A. \& Pinto, J. D., 2001. Phylogenetic studies of Meloidae (Coleoptera) with emphasis on the evolution of phoresy. Systematic Entomology, 26: 33 72 .

Bologna, M. A. \& Pinto, J. D., 2002. The Old World genera of Meloidae (Coleoptera): a key and synopsis. Journal of Natural History, 36: 2013-2102.

Borchmann, F., 1917. Pars 69. Meloidae, Cephaloidae. In: E. Schenkling (ed.). Coleopterorum Catalogus auspiciis et auxilio W. Junk. Junk, Berlin. 208 pp.

CAPOTE, L. \& GARCíA-PARÍs, M., 2001. Distribución geográfica de los Cerocomini ibéricos (Coleoptera: Meloidae). Boletín de la Asociación Española de Entomología, 25:31-44.

CARDELÚs, B., 1987. Naturaleza ibérica. Radiotelevisión Española y Editorial Debate. Barcelona. $221 \mathrm{pp}$.

Champion, G. C., 1891. A list of the Heteromerous Coleoptera collected by Mr. J. J. Walker, R. N., F. L. $\mathrm{S}$., in the region of the Straits of Gibraltar, with descriptions of four new species. Transactions of the Entomological Society of London, 1891: 375-401.

Champion, G. C., 1902. An entomological excursion to Central Spain. Transactions of the Entomological Society of London, 1902: 115-129.

Champion, G. C., 1903. An entomological excursion to Bejar, Central Spain. Transactions of the Entomological Society of London, 1903: 165-182.

Champion, G. C., 1904. An entomological excursion to Moncayo, N. Spain. Transactions of the Entomological Society of London, 1904: 81-99.

CoBos, A., 1949. Datos para el catálogo de coleópteros de España, especies de los alrededores de Málaga. Boletín de la Real Sociedad Española de Historia Natural, 47: 563-599.

Cuní Martorell, M., 1881. Excursión entomológica y botánica a la Cerdaña española (Cataluña). Anales de la Sociedad Española de Historia Natural, 10: 367-389.

Cuní Martorell, M., 1888. Insectos observados en los alrededores de Barcelona. Anales de la Sociedad Española de Historia Natural, 17: 133-191.

Cuní Martorell, M., 1897. Fauna entomológica de la villa de Calella (Cataluña, provincia de Barcelona). Anales de la Sociedad Española de Historia Natural, 26: 281-339.

Cuní Martorell, M. \& Martorell Peña, M., 1876. Catálogo metódico y razonado de los coleópteros observados en Cataluña. Gorchs. Barcelona. 360 pp.

DIECK, G., 1870. Eine entomologische Wintercampagne in Spanien. Berliner entomologische Zeitschrift, 14: 145-184.

EsCHERICH, K., 1896. Meloiden-studien. IV Theil. Wiener Entomologische Zeitung, 15: 27-30.

FLACH, K., 1907. Beiträge zur Käferfauna der Iberische Halbinsel. Wiener Entomologische Zeitung, 26: 17-19.
Fuente, J. M. DE LA, 1927. Lista sistemática de los coleópteros enviados por el P. Navás en Junio de 1926 según el Catálogo de 1906. Boletín de la Sociedad Entomológica de España, 10: 30-33.

FuENTE, J. M. DE LA, 1933. Catálogo sistemático geográfico de los Coleópteros observados en la Península Ibérica, Pirineos propiamente dichos y Baleares. Boletín de la Sociedad Entomológica de España, 16: 18-49.

GARCÍA-PARÍs, M., 1998. Revisión del género Berberomeloe Bologna, 1988 (Coleoptera: Meloidae) y diagnosis de un endemismo ibérico olvidado. Graellsia, 54: 97-109.

GARcíA-PARÍs, M., 2000. Nuevas localidades de Sitarini (Col. Meloidae) de la Península ibérica. Boletín de la Asociación Española de Entomología, 24: 197-200.

García-París, M., Ruiz, J. L. \& Martínez-Solano, I., 1999. Primeros datos sobre la zona de contacto entre Berberomeloe insignis (Charpentier, 1818) y $B$. majalis (Linnaeus, 1758) en Almería (Coleoptera: Meloidae). Graellsia, 55: 223-224.

GóRRIZ MuÑoz, R. J., 1881. Nuevas observaciones sobre costumbres y metamorfosis de algunos vesicantes. Actas de la Sociedad Española de Historia natural, 10: $59-64$.

GÓRRIZ MUÑOZ, R. J., 1882. Ensayo para la monografía de los coleópteros melóidos indígenas con aplicación a las ciencias médicas. Imprenta de J. Sanz, Zaragoza. 199 pp., 2 láms.

GóRriz MuÑoz, R. J., 1902. Coleópteros de la Cuenca del Ebro. Boletín de la Sociedad Aragonesa de Ciencias Naturales, 1: 180-186.

IgLESIAS, L. 1928. Notas Entomolóxicas. Insectos de Galiza. Nós, 53: 89-94.

Jeremías, X., Escolá, O. \& Pérez de Gregorio, J. J., 2002. Cerocomini ibéricos (Coleoptera: Meloidae), nuevos datos sobre su distribución geográfica en Cataluña (NE de la península ibérica). Boletín de la Asociación Española de Entomología, 26: 9-12.

KASZAB, Z., 1951. Über die arten der gattung Oenas. Acta Biologica Academiae Scientiarum Hungaricae, 2: 275-279.

KüSter, H. C., 1847. Die Käfer Europas. 12 heft. Baner \& Raspe. Nürnberg. pp. 83-84.

Laguna, M. A., 1902. Coleópteros recogidos durante el mes de Marzo. Boletín de la Sociedad Aragonesa de Ciencias Naturales, 1: 76-78.

LuCAS, P., 1849. Exploration scientifique de l'Algérie pendant les années 1840, 1841, 1842, publiée par ordre du Gouvernement et avec le concours d'une commission. Historie Naturelle des Animaux Articulés. II. Coléoptères. Imprimérie Nationale. Paris. 590 pp. 47 tab.

MAder, L., 1927. Meloidae. In: Winkler, A. (ed.). Catalogus Coleopterorum Regiones Palaearcticae, vol. II. Winkler. Wien: 851-888. 
Martínez SÁEZ, F. P., 1873. Datos sobre algunos coleópteros de los alrededores de Cuenca. Anales de la Sociedad Española de Historia Natural, 2: 53-75.

MedinA, M., 1895. Coleópteros de Andalucía existentes en el Museo de Historia Natural de la Universidad de Sevilla, clasificados por Dr. Francisco de P. Martínez y Sáez. Anales de la Sociedad Española de Historia Natural, 2: 53-75.

Melic, A. \& Blasco-Zumeta, J., 1999. Inventario provisional de la Biodiversidad monegrina (31 de Marzo de 1999). Boletín de la Sociedad Entomológica Aragonesa, [1998], 24: 215-251.

MitTer, H., 1984. Beitrag zur coleopterenfauna von Albarracin und umgebung (Provinz Teruel, Spanien). Linzer biologische Beiträge, 16: 181-193.

NAVÁs, L., 1904. Notas zoológicas, V. Excursión al Moncayo. Boletín de la Sociedad Aragonesa de Ciencias Naturales, 3: 139-167.

OliveirA, M. P. DE, 1894. Catalogue des Insectes du Portugal. Coléoptères. Impresa da Universidade. Coimbra. 393 pp.

Olivier, A. G., 1792. Encyclopèdie mèthodique. Dictionnaire des Insectes, vol. 7. Panckoucke. Paris. $827 \mathrm{pp}$.

Ortuño, V. M. \& Hernández, J. M., 1993. Diversos casos teratológicos en Coleoptera. Boletín de la Real Sociedad Española de Historia Natural (Sección Biológica), 89: 163-179.

Pardo Alcaide, A., 1949. Estudios sobre Meloidae II. Los Mylabrini de la Península Ibérica. Boletín de Patología Vegetal y Entomología Agrícola, 17: 61-82.

Pardo Alcaide, A., 1950 Los géneros de Meloidae de la fauna hespérica. Graellsia, 8: 39-79.

Pardo Alcaide, A., 1956. Los Cerocoma de la fauna ibérica (Col. Meloidae). Graellsia, 14: 21-27.

Pardo Alcaide, A., 1958. Los Sitarini de la Península Ibérica (Col. Meloidae). Graellsia, 16: 13-22.

Pérez Moreno, I. \& CABrero SAÑudo, F. J., 1999. Datos para el catálogo de coleópteros de La Rioja (Insecta, Coleoptera). Zubía, 11: 93-126.

Pinto J. D. \& Selander, R. B., 1970. The bionomics of the blister beetles of the genus Meloe and a classification of the New World species. Illinois Biological Monographs, 42: 1-222.

Recalde, J. I., San Martín, A. F. \& Pérez-Moreno, J., 2002. Meloidae. Catalogus Entomofauna Aragonesa, 26: 3-21.

Redondo, A., 1915. Coleópteros de Salamanca. Brotéria (Serie Zoologica), 1915: 14-48.

ReITTER, E., 1895. Bestimmungs-Tabellen der europaïschen Coleopteren Meloidae. I Theil: Meloini. 32. Ver, Verfassers. Paskau: 1-13.
Rivas Mateos, 1897. Una excursión á la Sierra de Béjar (provincias de Cáceres, Salamanca y Ávila). Actas de la Sociedad Española de Historia Natural, 26: 204210.

RodríGUEZ LÓPEZ-NeYRA, C., 1914 Claves dicotómicas para la determinación de los meloideos indígenas. Boletín de la Real Sociedad Española de Historia Natural, 14: 461-475.

Rosenhauer, W. G., 1856. Die Thiere Andalusiens nach dem Resultate einer Reise zusammengesetellt. Theodor Blaesing. Ernlangen. 429 pp.

RuIz, J. L., 1996. Sitarobrachys thoracica (Kraatz, 1862), nuevo género y especie para la coleopterofauna ibérica (Coleoptera, Meloidae). Zoologia Baetica, 7: 53-69.

RuIZ, J. L., 2001. Las Stenoria Mulsant, 1857 del grupo de $S$. apicalis (Latreille, 1802) de la Península Ibérica (Coleoptera, Meloidae). Graellsia, 57(2): 161-173.

Ruiz, J. L. \& ÁviLA, J. M., 1993. Contribución al conocimiento de los Meloidae (Coleoptera) en el sur de la Península Ibérica. Boletín de la Asociación Española de Entomología, 17: 141-148.

Ruiz, J. L., Ávila, J. M. \& Salado, R., 1993. Confirmación de la presencia de Meloe (Eurymeloe) baudueri Grenier, 1863 en la Península Ibérica. Boletín de la Asociación Española de Entomología, 17(1): 203.

Ruiz, J. L., SÁnchez-Piñero, F. \& Ávila, J. M., 1994a. Faunística y corología de los Meloidae (Coleoptera) de zonas áridas del sureste de la Península Ibérica. Boletim da Sociedade Portuguesa de Entomologia, 2(supl. 3) (Actas V Congr. Ibér. Entomol.) (1992): 325-335.

Ruiz, J. L., SÁNChez-Piñero, F. \& Ávila, J. M., 1994b. Sobre la presencia de Meloe (Taphromeloe) foveolatus Guérin de Méneville, 1842 en la Península Ibérica (Coleoptera: Meloidae). Boletín de la Asociación Española de Entomología, 18(3-4): 104.

SAlvaÑá Comas, J. M., 1870. Apuntes para la geografía y fauna entomológicas de Mataró. Imprenta de Gregorio Juste. Madrid. 44 pp.

Serrano, A. R. M., 1981. Contribuição para o estudo dos coleópteros do Parque Natural da Arrábida. Parques Naturais, 9. Serviço Nacional de Parques, Reserrvas e Património Paisagistico. Lisboa. 87 pp.

SERrano, A. R. M., 1983. Contribuição para o inventário dos coleópteros em Portugal. Instituto Nacional de Investigaçao Agrária. Estação Agronómica Nacional. Oeiras. 269 pp.

Sumacov, G., 1934. Inventa entomologica itineris hispanici et maroccani, quod a 1926 fecerunt Harald et Hakan Lindberg. XXI. Méloides du Bassin Méditerranéen et du Maroc. Commentationes Biologicae, 4(13): 1-6. 
Torres SAla, J., 1962. Catálogo de la colección entomológica "Torres Sala" de coleópteros y lepidópteros de todo el Mundo. I. Instituto Alfonso El Magnánimo. Diputación Provincial de Valencia. Valencia. 487 pp.

Traizet, E., 1896. Observations biologiques sur le Notoxus monoceros (Col.). Bulletin de la Societé Entomologique de France, 1896: 104. (fide Bologna \& Aloisi, 1994. Obra original no consultada).

Trotta-Moreu, N. \& García-París, M., 2001. Los Hycleus (Coleoptera: Meloidae) de la Península Ibérica: distribución geográfica y variabilidad elitral. Graellsia, 57: 99-111.
UhAGÓN, S., 1887. Coleópteros de Badajoz. Tercera Parte. Anales de la Sociedad Española de Historia Natural, 16: 373-380.

Valladares Díez, L. F., 1984. Estudio de los coleópteros vesicantes de la provincia de León (Coleoptera, Meloidae). Celarayn. León. 156 pp.

Valladares, L. F. \& Salgado, J. M., 1983. Contribución al conocimiento de los Meloidae (Col.) en la provincia de León. Boletín de la Asociación Española de Entomología, 7: 79-100. 